\title{
Review of Contributions to the Workshop on SN1993J
}

\author{
By J. CR A I G WHEELER \\ AND ALEXEI V. FILIPPENK O \\ ${ }^{1}$ Department of Astronomy, University of Texas, Austin, TX 78712, USA \\ ${ }^{2}$ Department of Astronomy and Center for Particle Astrophysics, University of California, \\ Berkeley, CA 94720, USA
}

At its peak, SN 1993J was one of the brightest supernovae in this century, and it is being studied more thoroughly than any supernova except SN 1987A. It is proving to be similar to the transition object SN 1987K, which metamorphosed from being a hydrogen-rich Type II near peak to having a hydrogen-deficient nebular phase. SN 1993J has been observed throughout the electromagnetic spectrum and with optical spectropolarimetry. It is interacting with a dense circumstellar nebula and is generating radio and X-ray flux, but it has probably not been detected in gamma rays. The photometric and spectral evolution are consistent with a star of original mass $\sim 15 \mathrm{M}_{\odot}$ that lost appreciable mass to a binary companion leaving an extended, helium-rich hydrogen envelope of $\$ 0.5 \mathrm{M}_{\odot}$ and a helium core of $\sim 4 \mathrm{M}_{\odot}$. The spectral evolution will put strong constraints on the mixing of ${ }^{56} \mathrm{Ni}$ and other species.

\section{Introduction}

SN 1993J was discovered on March 28.9 by F. Garcia (Ripero 1993) in the Sab galaxy NGC $3031=$ M81. It was the brightest supernova observable from mid-northern latitudes since SN 1972E and has been the subject of intense observation by a large number of major and minor optical observatories, the VLA and other radio telescopes, IUE, the Compton Gamma Ray Observatory, ROSAT, and the newly launched ASCA satellite, as well as by a host of amateur astronomers. In addition, SN 1993J has proven exceptional on a number of grounds and has prompted considerable theoretical modeling. IAU Colloquium 145 provided the first opportunity for a public exchange on the early observational and theoretical results. These were presented in a workshop organized and chaired by one of us (AVF), and in an associated poster session. The organizing committee of the Colloquium determined that a synthesis of the results was preferable to a collection of small individual contributions. This review is the outcome.

\section{Prediscovery Observations}

Prediscovery observations put useful limits on the progenitor magnitude (Perelmuter 1993; Humphreys et al. 1993; Salzer et al. 1993; Prugniel 1993). The image is extended slightly in the north/south direction (Blakeslee and Tonry 1993). It is superposed on a ridge of diffuse starlight, about $3^{\prime \prime}$ wide and $20^{\prime \prime}$ long at position angle $40^{\circ}$, that is somewhat stronger in $B$ than in $\mathrm{H} \alpha$ (Tomaney and Crotts 1993) and shows no H II region within $10^{\prime \prime}$ of the supernova (Richmond 1993). There were some early reports of discrepant magnitudes in the IAU Circulars, raising the issue of variability, but no further evidence for such variation has emerged. This may have represented some calibration differences, but a physical variation cannot be ruled out. Filippenko (1993a) pointed out that the colors and magnitudes given by Perelmuter (1993) are consistent with those of a K0 Ia supergiant. 
Table 1. Progenitor Magnitudes

\begin{tabular}{ccccccc}
\hline \hline & $U$ & $B$ & $V$ & $R_{c}$ & $I_{c}$ \\
\hline$m^{1}$ & 21.45 & 21.73 & 20.72 & 19.94 & 19.43 \\
$M^{2}$ & -7.0 & -6.6 & -7.5 & -8.2 & -8.6 \\
\hline
\end{tabular}

${ }^{1}$ Aldering et al. (1993), without Petermuter (1993)

${ }^{2}$ Assuming $E(B-V)=0.15 \mathrm{mag}$ and $\mathrm{DM}=27.7$; de Vaucouleurs (1993).

The image can be separated into two components, with the brighter corresponding to the position of the supernova. Table 1 gives revised colors of this component from Aldering et al. (1993). Humphreys et al. (1993), Aldering et al. (1993), and Podsiadlowski et al. (1993) concluded that the colors require the presence of at least two photometric components within the brighter image. One component dominates in the $U$ and $B$ bands; it could be a late B to early A supergiant or an OB association. The other component dominates in the $R$ and $I$ bands in a manner consistent with a late $\mathrm{G}$ to early $\mathrm{K}$ supergiant. The bolometric magnitudes are in the range -7 to -8 with the best fit about -7.8 for a distance modulus (DM) of $27.7 \mathrm{mag}$ and $A_{V}=0.5 \mathrm{mag}$ (Aldering et al. 1993). This marginally excludes an AGB star progenitor with maximum bolometric luminosity of $-7.1 \mathrm{mag}$ (but see Blöcker and Schönberner 1991; Hashimoto, Iwamoto, and Nomoto 1993). The corresponding main sequence mass range is $15-20 \mathrm{M}_{\odot}$ with a best estimate of $\sim 17 \mathrm{M}_{\odot}$ (Aldering et al. 1993).

Care must be taken in interpreting these observations. At the distance of M81 an arc second corresponds to about 17 parsecs, and one could easily be looking at an association or a cluster. There is some temptation to identify the red component of the blended image with the progenitor, but the only sure way to test for the progenitor is to wait until the supernova fades. Podsiadlowski et al. (1993) point out that in the binary transfer scenario, the mass gaining companion could be brighter than the progenitor, leaving open the possibility that neither of the two stars arising in the photometric fits represents the progenitor. Nevertheless, some models for the progenitor predict a star with about the right colors and luminosity of the red component of the pre-discovery images, and it is certainly possible that the progenitor was observed.

\section{Outburst and Reddening}

Wheeler et al. (1993) reported that the shock outbreak is estimated to have occurred at JD 2449074.5 \pm 0.1 (March 28.0 $\pm 0.1 \mathrm{UT}$ ) and thus is defined to within a few hours. De Vaucouleurs (1993) has revised this to JD 2449074.7 \pm 0.1 (March $28.2 \pm 0.1$ ). Wheeler et al. estimated the color excess to be $E(B-V) \leq 0.3 \mathrm{mag}$ in order not to violate the limiting value of $B-V$ for high temperatures, and $E(B-V)=0.15 \pm 0.02 \mathrm{mag}$ from examinations of residuals to black body fits to spectra on March 31 and April 1 and from $U B V$ colors. Wamsteker et al. (1993) estimate $E(B-V)=0.07$ mag from the weakness of the $2200 \AA$ feature in IUE spectra. Freedman et al. (1994) derive a total mean extinction to thirty M81 Cepheids of $E(B-V)=0.11 \mathrm{mag}$. Thus, there is little direct support for the large values of $E(B-V) \approx 0.7$ mag reported by Humphreys $e t$ al. (1993).

Observations with the Carlsberg Automatic Meridian Circle on La Palma yielded an 


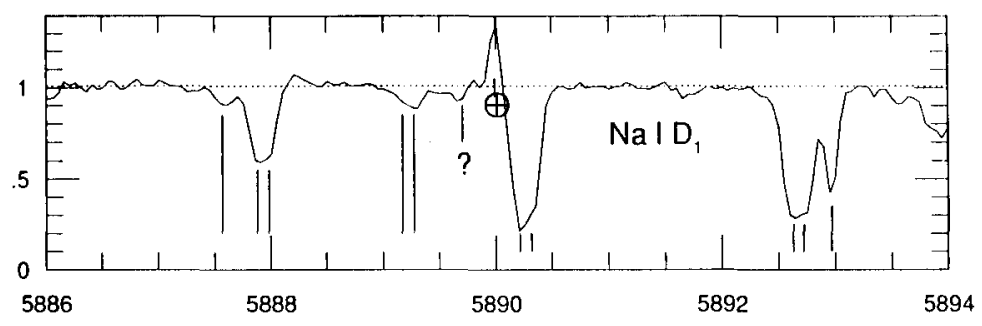

Wavelength $(\AA)$

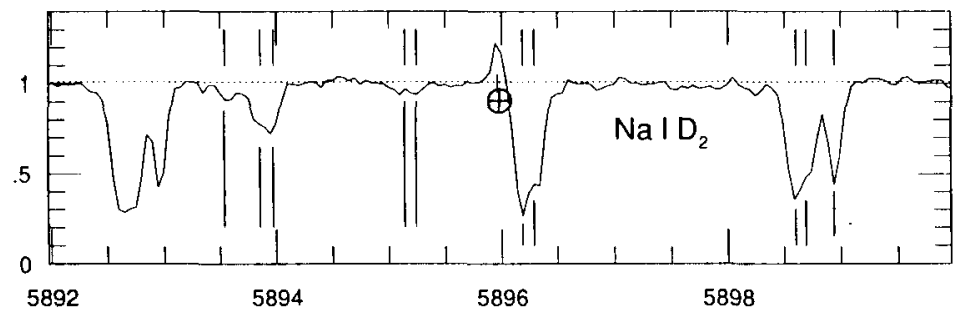

FIGURE 1. High-resolution spectra of the Na I D line. The top panel shows the shorter and the bottom panel the longer wavelength component shifted so that individual velocity components are aligned (Smith and Tomkin 1993).

optical position for the supernova of $\alpha=09^{h} 55^{m} 24.785^{s}, \delta=+69^{\circ} 01^{\prime} 13.69^{\prime \prime}$, equinox J2000.0 (Lewis et al. 1993). This position supersedes the one given by Morrison, Argyle, and Helmer (1993). A conservative estimate of the accuracy is $0.11^{\prime \prime}$ in $\alpha$ and $\delta$. This optical position is in excellent agreement with the VLA radio position (accuracy $0.2^{\prime \prime}$ in $\alpha$ and $\delta$; Van Dyk et al. 1993a).

\section{Interstellar Lines}

Meikle et al. (1993) report finding a minimum of 20 velocity components of the intervening interstellar medium in the $\mathrm{Na} I \mathrm{D}, \mathrm{Ca} \mathrm{II}$, and other narrow lines. Wheeler et al. (1993) give $-140,-125,-120,-61,-55,-6,0,+117,+122$, and $+134 \mathrm{~km} \mathrm{~s}^{-1}$ for ten of the strongest components of $\mathrm{Na}$ I D. These are illustrated in Figure 1. Wamsteker et al. (1993) identify interstellar lines of Si II + O I $\lambda 1300$, C II $\lambda 1335$, Si IV $\lambda 1400$, C IV $\lambda 1550$, Al II $\lambda 1671$, Fe II + Mn II $\lambda 2600, \mathrm{Mg}$ II $\lambda 2800$, and $\mathrm{Mg}$ I $\lambda 2852 \AA$ in IUE spectra. The $\mathrm{Mg} I$ line showed components at $-117,-63,+12,+129$, and $+181 \mathrm{~km} \mathrm{~s}^{-1}$. The largest negative components roughly correspond to the kinematics of interstellar H I features in M81 in the vicinity of the supernova (Rots and Shane 1975); they probably correspond to gas in the local disk. The features at $\sim-60 \mathrm{~km} \mathrm{~s}^{-1}$ are near the mean velocity of M81 of about $-49 \pm 10 \mathrm{~km} \mathrm{~s}^{-1}$ (de Vaucouleurs et al. 1991) and so may be gas in the halo of M81, but this component could also represent some local infall into the Galaxy. The very low velocity components are presumably from the local Galactic disk. The components at $\sim+120 \mathrm{~km} \mathrm{~s}^{-1}$ are especially interesting because they may represent flow within the M81-M82 group and also because they are considerably stronger than the components associated with M81 itself.

Benetti et al. (1993) and Wheeler et al. (1993) use the Na I column densities to estimate $E(B-V) \lesssim 0.4 \mathrm{mag}$, while Richmond et al. (1994) find $\sim 0.3 \mathrm{mag}$. 
TABLE 2. Revised Early Magnitudes ${ }^{1}$

\begin{tabular}{lll}
\hline \hline Observer & Epoch & Magnitude \\
\hline Merlin & March 27.91=JD 74.41 & $V \gtrsim 17$ \\
Neeley & March 28.3=JD 74.8 & $V=13.45 \pm 0.15$ \\
Garcia & March 28.91=JD 75.41 & $m_{V}=11.8$ \\
Rodriguez & March 28.93=JD 75.43 & $m_{C C D=11.4}$ \\
Garcia & March 28.98=JD 75.48 & $m_{V}=11.5$ \\
Ulfheden \& Hamberg & March 29.02=JD 75.52 & $m_{C C D=11.2}$ \\
Neeley & March 29.15=JD 75.65 & $V=11.05 \pm 0.2$ \\
Neeley & March 30.05=JD 76.55 & $V=10.68 \pm 0.2$ \\
\hline
\end{tabular}

${ }^{1}$ Visual and CCD data still provisional; de Vaucouleurs (1993b).

\section{Photometry}

$U B V R I$ photometry since March 30 was presented from the automatic imaging telescopes at Leuschner Observatory (Fig. 2; Filippenko et al. 1993b; Richmond et al. 1994), at the Kapteyn telescope (Lewis et al. 1993; Meikle et al. 1993), and by Wells et al. (1993) primarily from CCD imaging with the Case Western Reserve University Burrell Schmidt telescope at Kitt Peak National Observatory. See also van Driel et al. (1993).

Comparisons of star B with other field stars in the $V$ band indicate that it is not variable on short time scales as previously reported, but that one star is a newly discovered W UMa eclipsing binary. Photometry of the field stars has been evaluated by de Vaucouleurs, Corwin, and Skiff (1994). Table 2 gives the best current estimate of the prediscovery data after careful evaluation of filter responses, color corrections, etc. (de Vaucouleurs 1993; but see also Richmond et al. 1994). The $V$ light curve first peaked at $V=10.7 \mathrm{mag}$ (JD $76.8=$ March 30.3), 2.1 days after shock breakout. The color at that first peak was observed to be $B-V \lesssim 0.1 \mathrm{mag}$, with the corrected color being even slightly negative. The $U B V R I$ light curves all show a post-maximum dip, a second maximum, and a fairly rapid subsequent decline, followed by a more gradual tail. The intermediate minimum was 8.3 days after shock breakout (JD $82.8=$ April 5.3). The secondary $V$ maximum was attained on day 21.1 (JD 95.6 = April 18.1; April 15 in $U$; April 19 in $I$ ). On the tail, the slope in $V, R$, and $I$ is roughly $0.02 \mathrm{mag} \mathrm{d}^{-1}$, but in $B$ it has a substantially smaller slope $\left(\sim 0.01 \mathrm{mag} \mathrm{d}^{-1}\right)$. On May 2, the $U$ band decline leveled off as was seen in SN 1987A at day 125 (Suntzeff et al. 1988). Photometry from the week of June 3-10 indicates that $U$ is increasing at a rate of $0.0035 \mathrm{mag} \mathrm{d}^{-1}$. This may be consistent with the buried pulsar model (Ostriker 1987), as was suggested by Wheeler (1993).

The color curves displayed in Figure 3 show a very interesting evolution. The color is very blue at the first peak and reddens rapidly until the first minimum. The color reddens only slowly during the rise to second maximum, but then reddens rapidly once again beginning at the second peak, reaching a nearly constant plateau at the time, probably coincidentally, when the $V$ magnitude returns to the value of the first minimum. One may qualitatively understand the color evolution by interpreting the first reddening as the phase when the photosphere is in the outer, expanding hydrogen-rich layer, the flat portion when the light curve is on the second rise as the phase when the photosphere is in the inner, helium-rich layer, and the rapid post-peak reddening as the phase when the 


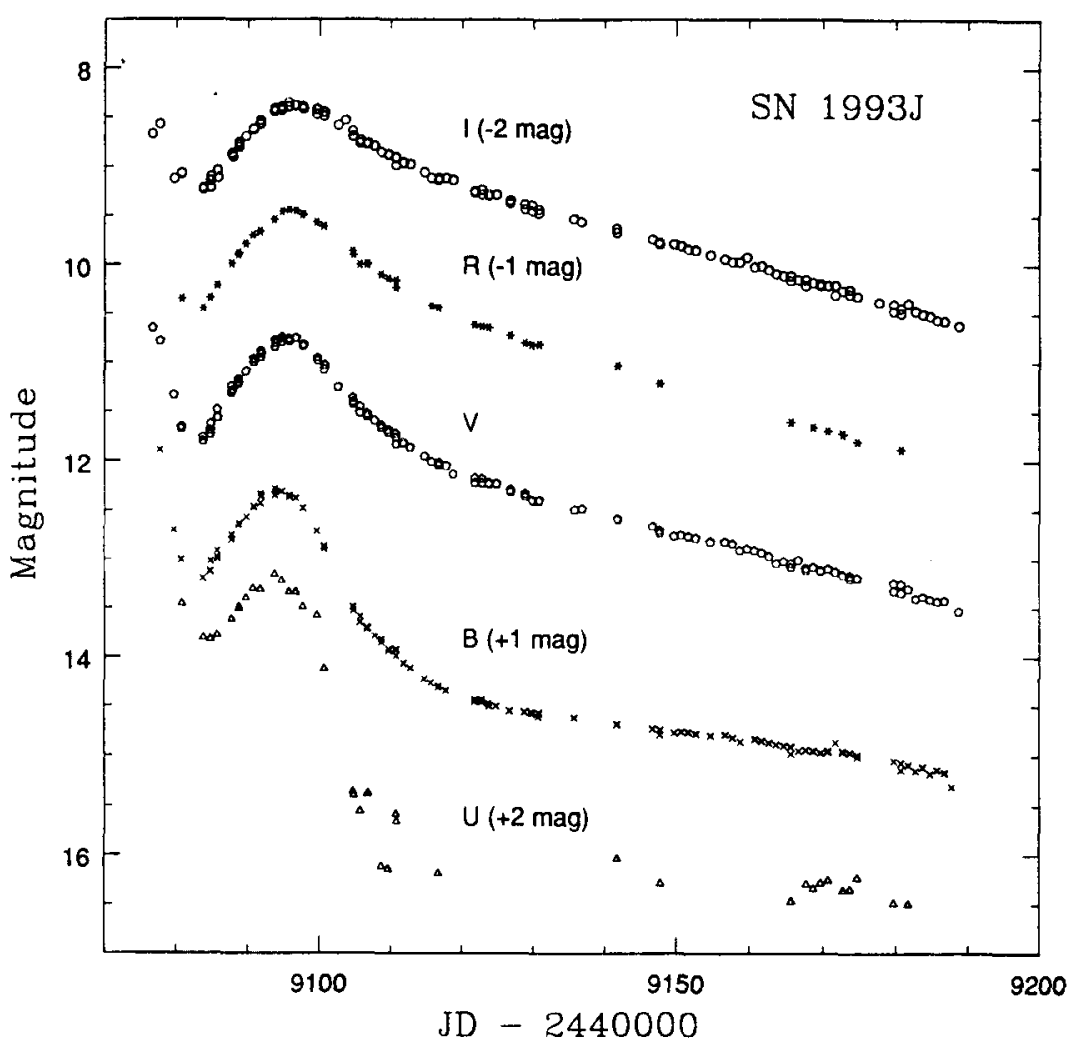

FIgURE 2. UBVRI photometry from Richmond et al. (1994); $0.76 \mathrm{~m}$ automatic imaging telescope at Leuschner Observatory.

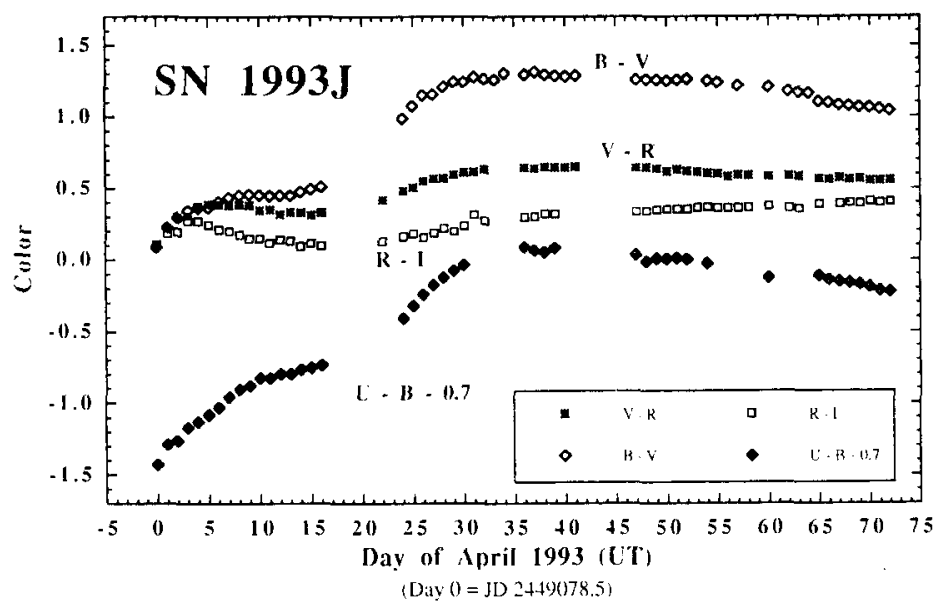

Figure 3. Color curves from Wells et al. (1993). 


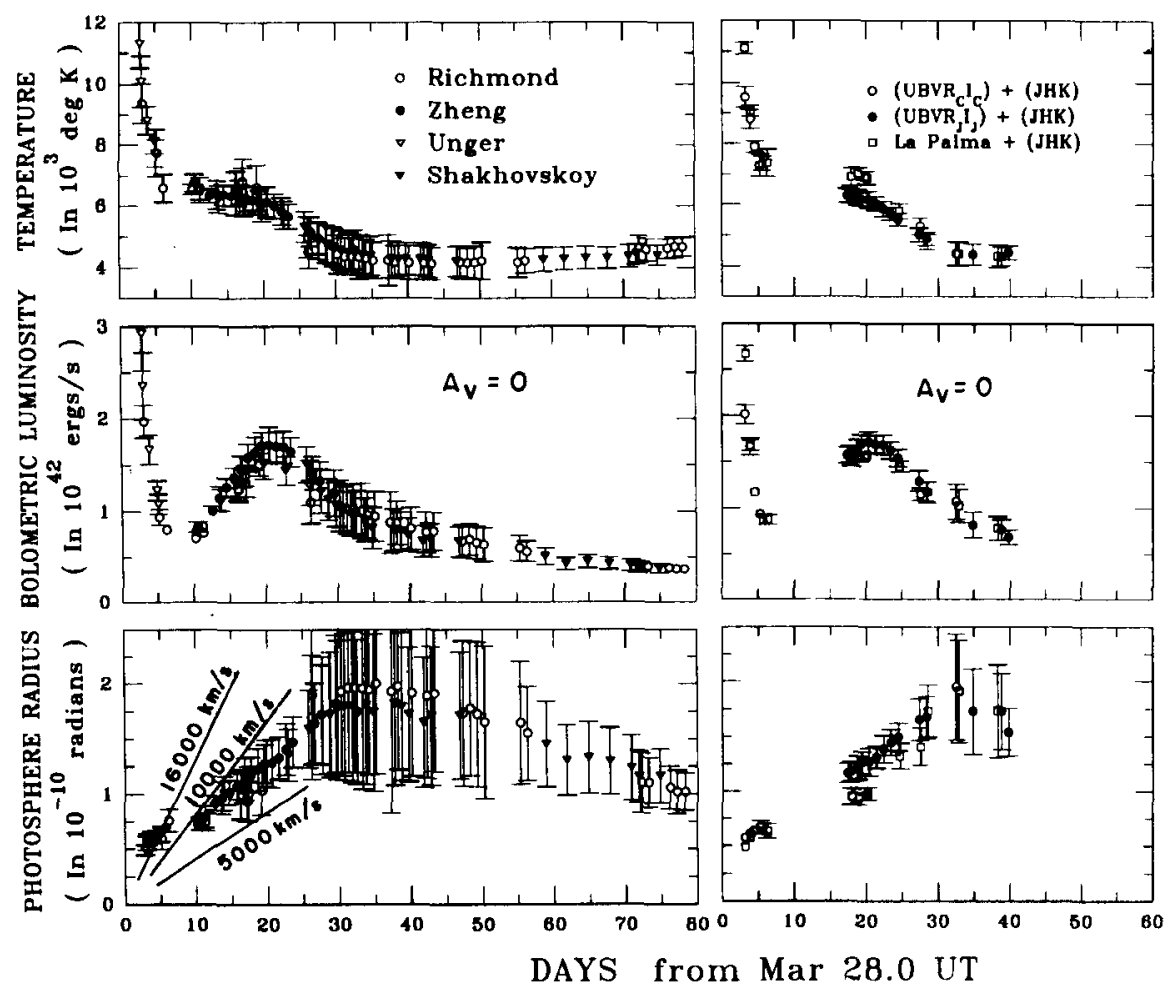

FiguRE 4. Bolometric luminosity, effective temperature, and photospheric radius versus time from Ray et al. (1993).

photosphere recedes into the inner, metal-rich core. The latter may yield lower temperatures because iron-peak elements are more efficient coolants than the overlying helium layer (Eastman 1993). If this is the proper interpretation, and that is not completely clear, it has important implications for the structure and composition of the ejecta, since theoretical models dictate different rates of evolution of the photosphere through different layers as a function of the mass of the core and the mixing of envelope hydrogen, freshly synthesized ${ }^{56} \mathrm{Ni}$, and other elements $(\S 12,13)$. It is important to understand whether this basic interpretation is also consistent with the observed spectral evolution to be described below.

Ray, Singh, and Sutaria (1993) used $U B V R I$ photometric data to determine a bolometric light curve and corresponding effective temperatures and radii of the photosphere, as given in Figure 4. The effective temperature has two plateaus: the first one at $\sim 6500 \mathrm{~K}$ extends between days 7 and 17 . The second is at about $4000 \mathrm{~K}$. The photospheric radius expands with a velocity around $16,000 \mathrm{~km} \mathrm{~s}^{-1}$ in the first few days; thereafter this velocity is between 7500 and $10,000 \mathrm{~km} \mathrm{~s}^{-1}$ (see bottom panels of Fig. 4), and the photosphere starts receding around day 40 .

\section{UV Spectroscopy}

Wamsteker et al. (1993) and Sonneborn et al. (1993) reported on IUE spectroscopy. The time of maximum UV flux was apparently earlier than March 30.2. The UV continuum flux at $1275,1735,2450$, and $2900 \AA$ was $126,115,75$, and 68 in units of $10^{-14} \mathrm{erg}$ 


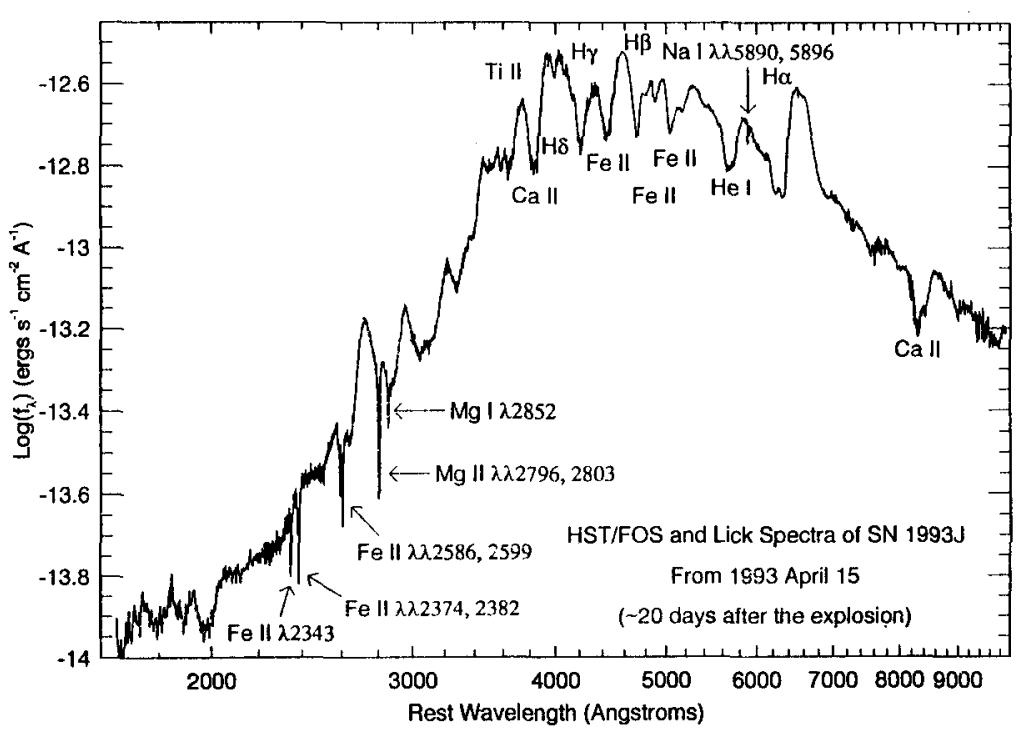

Figure 5. HST Faint Object Spectrograph UV spectrum of SN 1993J on April 15 along with a spectrum from Lick Observatory at nearly the same time (Jeffery et al. 1994). The spectrum has been corrected for extinction with $E(B-V)=0.1 \mathrm{mag}$.

$\mathrm{s}^{-1} \mathrm{~cm}^{-2} \AA^{-1}$ on March 30.2 and decreased by factors of $203,83,29$, and 11 , respectively, between March 30.2 and April 4.6, with the rate of decline slowing after April 2. The spectrum showed few features in the early epochs except for interstellar features and $\mathrm{N} \mathrm{V} \lambda \lambda 1238.8,1242.8 \AA$. The $1238.8 \AA$ line had FWHM $<50 \mathrm{~km} \mathrm{~s}^{-1}$ and the $1242.8 \AA$ component a red wing extending $>200 \mathrm{~km} \mathrm{~s}^{-1}$ from line center. The $\mathrm{N} \mathrm{V}$ integrated line flux was $3.9 \times 10^{-12} \mathrm{erg} \mathrm{cm}^{-2} \mathrm{~s}^{-1}$ on March 30.2 and decreased by a factor of 43 by April 4.6. The narrow width, large flux, prompt appearance, and rapid decay suggest that this emission arises in slowly moving, dense material close to the progenitor, most likely a stellar wind, characteristic of late-type supergiants. The high state of ionization strongly indicates that the wind has been ionized and heated by the UV and X-rays from the shock breakout as well as the radiation resulting from the interaction of the ejecta and circumstellar medium (Lundqvist, Fransson, and Chevalier 1993).

SN 1993J was observed on April 15 with the Hubble Space Telescope. A preliminary reduction of the data is shown in Figure 5 (Jeffery et al. 1994) along with a nearly simultaneous optical spectrum obtained by A.V. Filippenko and T. Matheson at Lick Observatory. The spectrum continues to show a definite UV deficit with respect to a Planckian at this epoch.

\section{Optical Circumstellar Lines}

In addition to the broad features, SN 1993J exhibited several narrow, unresolved (FWHM $\lesssim 5 \AA$ ) emission lines in the earliest spectra that faded rapidly. La Palma spectra from March 29.9 to April 4.9 are shown in Figure 6. Spectra from Lick and La Palma observatories revealed $\mathrm{H} \alpha$, He II $\lambda 4686$, [Fe X] $\lambda 6374$, [Fe XI] $\lambda 7892$, and [Fe XIV] $\lambda 5303$ (see also Andrillat 1993; Benetti et al. 1993). Benetti et al. (1993) give the centroid of the $\mathrm{H} \alpha$ emission at $6561.4 \AA$, yielding a heliocentric velocity of $-82 \mathrm{~km} \mathrm{~s}^{-1}$. Note that this does not correspond to any of the interstellar velocities. The line is resolved 


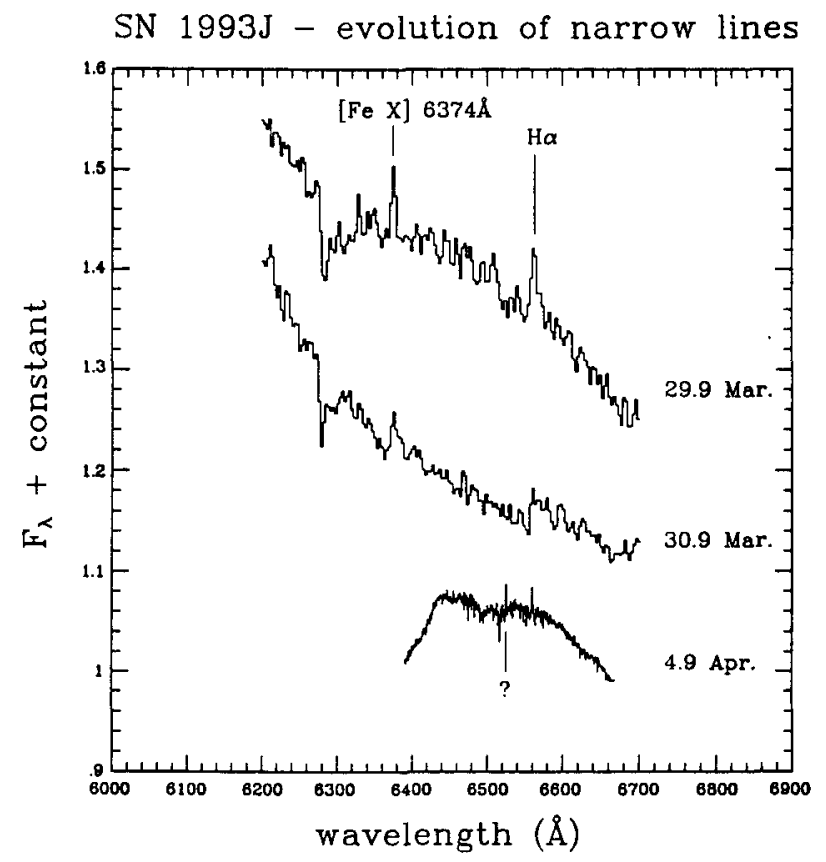

Figure 6. Evolution of the narrow circumstellar lines (Lewis et al. 1993; Meikle et al. 1993).

with a FWHM of $4.5 \AA\left(200 \mathrm{~km} \mathrm{~s}^{-1}\right)$ and an equivalent width (EW) of $0.34 \AA$. The March La Palma spectra were taken at a resolution of $6 \AA$. The $\mathrm{H} \alpha$ line is just resolved, with a FWHM of $8 \AA$. The iron lines are unresolved. A higher resolution $(R=0.82 \AA)$ spectrum on April 4 shows that $\mathrm{H} \alpha$ was still present, though much weaker, and with a FWHM of $2 \AA$. The measured intensities of these lines are shown in Table 3. The H $\alpha$ line declined roughly exponentially with an $e$-folding time of just over 2 days.

The narrow lines indicate the presence of circumstellar material around the progenitor. When the supernova shock wave emerged from the photosphere, the resulting flash of UV and X-rays would have ionized the dense, slow-moving wind of the progenitor which then recombined, producing the narrow lines. The presence of circumstellar material is also supported by the appearance of radio and X-rays, produced when the fast-moving ejecta plowed into the wind $(\S 14,15)$, and this may have been responsible for the rapid fading of the narrow lines.

\section{Low Dispersion Optical Spectra}

Early optical spectra have been reported by Garnavich and Ann (1993), Filippenko, Matheson, and Ho (1993a), Liebert (1993), Lewis et al. (1993), Schmidt et al. (1993), Wheeler et al. (1993), $\mathrm{Hu}$ et al. (1993), and other groups. A series of spectra covering the span from March 29 to May 31 from La Palma is shown in Figure 7. They have not been dereddened.

The first spectrum on record seems to be that from La Palma on March 29.9. The early spectra are nearly featureless on large scales, but contain several broad peaks and valleys with relative amplitudes of a few percent (e.g., Filippenko and Matheson 1993a; Taniguchi et al. 1993). The exact profiles of these subtle features are affected by telluric lines, and various detector artifacts in some data. A wide slit helps avoid losses due to 
TABLE 3. Circumstellar line fluxes and upper limits

$$
\left(10^{-14} \mathrm{erg} \mathrm{s}^{-1} \mathrm{~cm}^{-2}\right)
$$

(Lewis et al. 1993; Meikle et al. 1993).

\begin{tabular}{lllllll}
\hline \hline line: & $\mathrm{H} \alpha$ & $\mathrm{He} \mathrm{II}$ & {$[\mathrm{Fe} \mathrm{X}]$} & {$[\mathrm{Fe} \mathrm{XIV]}$} & {$[\mathrm{Fe} \mathrm{XI}]$} \\
$\lambda_{0}(\AA)$ & 6563 & 4686 & 6374 & 5303 & 7892 \\
\hline Mar 29.875 & 6.7 & - & 3.9 & $<3.0$ & 0.5 \\
Mar 30.875 & 4.4 & $<6.5$ & 2.5 & $<2.5$ & $<0.4$ \\
Apr 01.06 & $<2$ & $<3$ & $<1$ & $<1$ & $<0.15$ \\
Apr 04.87 & 0.46 & - & - & - & - \\
\hline
\end{tabular}
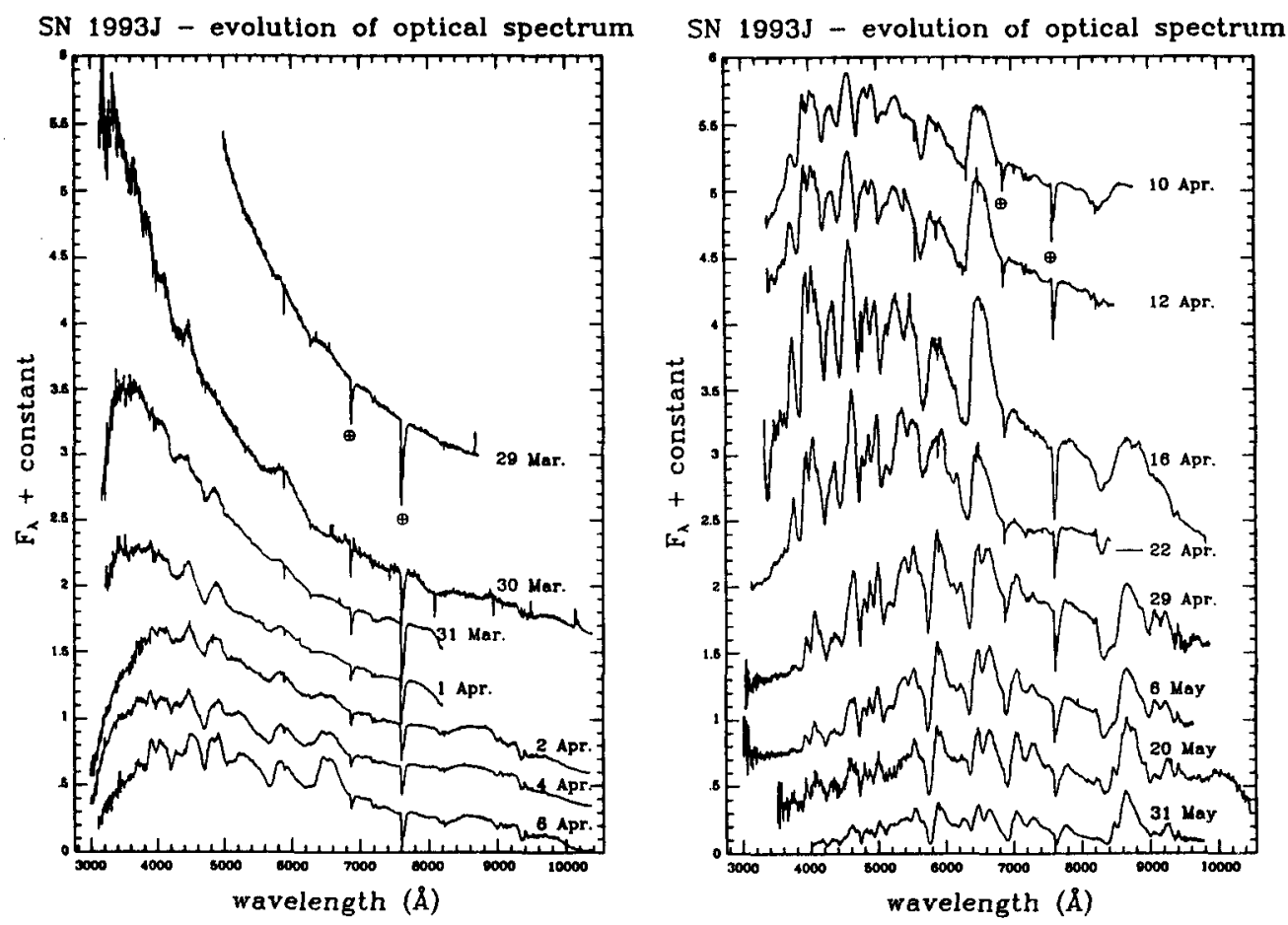

Figure 7. Evolution of the optical spectra (Lewis et al. 1993; Meikle et al. 1993).

atmospheric dispersion. The continuum slopes in the data of Garnavich and Ann (1993) may be affected in this way.

The early continua seem to be well defined black bodies. On March 30, Meikle et al. (1993) give a best fit temperature of $16,000 \pm 1000 \mathrm{~K}$ with an adopted $A_{V}$ of 0.25 mag. Wheeler et al. (1993) give $T=14,900 \pm 1000 \mathrm{~K}$ on March 31.18 and $T=11,800 \pm 700 \mathrm{~K}$ on April 1.14, and simultaneously a value of $E(B-V)=0.15 \pm 0.02 \mathrm{mag}$.

The emission feature at $6450 \AA$ has a corresponding absorption trough with a minimum 
at $6270 \AA$. If this is $\mathrm{H} \alpha$, the expansion velocity derived from the minimum of the trough would be $13,000 \mathrm{~km} \mathrm{~s}^{-1}$, with an emission peak blueshifted by $5000 \mathrm{~km} \mathrm{~s}^{-1}$ (Filippenko and Matheson 1993a). There is, however, a weak telluric line at $6280 \AA$ that partially obscures the true line profile at this early epoch. The $\mathrm{H} \alpha$ emission is still subtle on April 2, but distinct by April 5 at the first visual minimum. The emission profile is rather unique. Even at this very early stage it is flat-topped with two small emission components or a small absorption dip.

The La Palma spectrum from March 30 (Fig. 7) shows a broad emission peak at about $5900 \AA$ that may be He I $\lambda 5876$. This peak fades perceptibly the next night and is joined with a broad absorption to the blue on April 1, which is quite definite by April 4. Whether it is He I $\lambda 5876, \mathrm{Na}$ I D, or a combination is not clear. By the end of March, $\mathrm{H} \beta$ and $\mathrm{H} \gamma$ P-Cygni lines were clearly present, indicating that SN 1993J was a Type II event. Their profiles show material moving at least as fast as $15,000 \mathrm{~km} \mathrm{~s}^{-1}$. Wheeler et al. (1993) report shallow P-Cygni $\mathrm{H} \beta$ with minimum at $\sim 4680 \AA$ corresponding to a velocity of $11,200 \mathrm{~km} \mathrm{~s}^{-1}$ on March 31 - April 1, and P-Cygni He I $\lambda 5876$ on April 1 with an absorption minimum at $\sim 5593 \AA$ corresponding to a velocity of $14,500 \mathrm{~km} \mathrm{~s}^{-1}$.

In most data sets, the $\mathrm{H} \alpha$ profile remains basically flat-topped until the strong $\mathrm{He} I$ lines intrude around April 25. The La Palma data from April 12, 16, and 22 exhibit a more single-peaked profile with the April 22 data, in particular, showing a central narrow spike that does not seem to appear in other data from nearly the same epoch. A Lick spectrum from April 13 (see Fig. 14 below) shows a similar spike, whereas La Palma spectra on April 12 and 16 show some structure, but not this same spike. This behavior may represent transient emission features as the shock overtakes lumps in the circumstellar matter.

\section{The Helium Transition}

The character of the optical spectra changed in a dramatic way shortly after April 22 , about 25 days after outburst. The most distinct alteration was the "double-peaked" nature of the $\mathrm{H} \alpha$ line ( $\mathrm{Hu}$ et al. 1993). This feature can be recognized (with hindsight) in the April 24 spectrum of Schmidt et al. (1993). There was early speculation that this represented some grossly non-spherical flow, but this was clarified by the recognition by Filippenko and Matheson (1993b; see also Filippenko, Matheson, and Ho 1993a) that the spectrum was showing strong lines of $\mathrm{He} \mathrm{I}$, and that the second peak in $\mathrm{H} \alpha$ was $\mathrm{He} \mathrm{I}$ $\lambda 6678$. With this recognition, there is little, if any, direct evidence for asymmetry in the spectra (but see $\S 11$ for the spectropolarimetry).

There are some earlier hints for this double peaked nature of $\mathrm{H} \alpha$ in the April 6 spectrum of Figure 7, in the April 7 spectrum from the Perkins $1.8 \mathrm{~m}$ telescope (Baron et al. 1993), and in unpublished spectra from McDonald Observatory on April 5, near the first minimum. Whether this is an early emergence of He I which then fades before its later strong reemergence is a question that needs to be investigated.

Figure 8 illustrates this transition to strong He lines. Note the second peak next to $\mathrm{H} \alpha$ due to $\mathrm{He} \mathrm{I} \lambda 6678$, the new absorption corresponding to He I $\lambda 7065$, and the change in the line profile as He I $\lambda 5876$ induces a sharp absorption at $5700 \AA$.

\section{IR Spectroscopy}

Infrared spectroscopy was conducted at McDonald Observatory on the decline from the first peak. The spectra in the $J$ and $K$ bands on March 31.2 showed only a continuum within the limits of the noise. Spectra on April 1.2 and 2.2 showed definite evidence for 


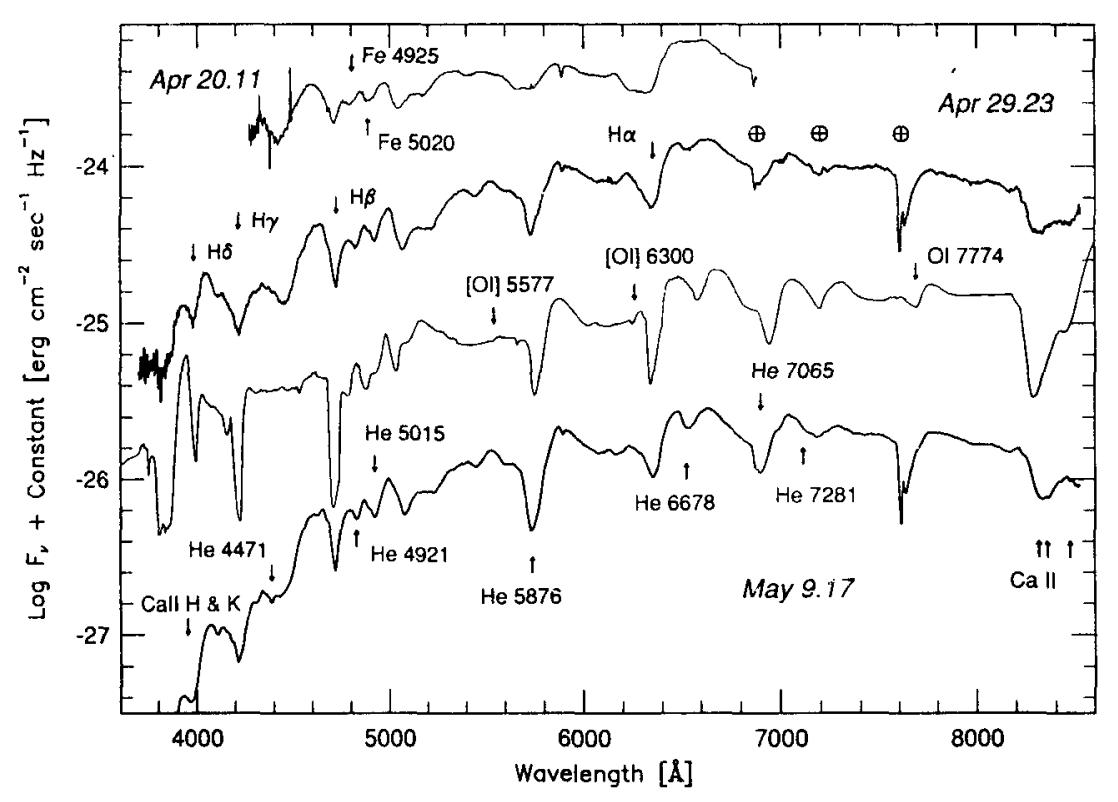

Figure 8. Optical spectra from April 20.11, 29.23, and May 9.17 showing the onset of the strong helium lines and a model atmosphere spectrum (Swartz et al. 1993b; $§ 13$ ).

$\mathrm{Pa} \beta 1.282 \mu \mathrm{m}$ as a broad emission bump centered at $1.2874 \mu \mathrm{m}$ with a total velocity width of $v \approx 11,000 \mathrm{~km} \mathrm{~s}^{-1}$ (Swartz et al. 1993b). The IR data of that epoch show no sign of $\mathrm{He} \mathrm{I}$ or other $\mathrm{H}$ lines.

IR spectra from May 3.16, after the onset of the He I lines in the optical, show the 1.083 and $2.058 \mu \mathrm{m}$ lines of He I, as seen in Figure 9. The former exhibits a P-Cygni profile and the latter an asymmetric absorption with only a broad, low amplitude emission excess to the blue. There was little emission in this line in SN $1987 \mathrm{~A}$, but the reasons are unknown. These data also show a P-Cygni profile of $\mathrm{Pa} \beta$, perhaps flat-topped, and maybe $\mathrm{Ca}$ II at $1.195 \mu \mathrm{m}$, but this identification is uncertain. $\mathrm{Br} \gamma$ may be present, but is not pronounced. The $K$-band spectrum is essentially featureless.

\section{Spectropolarimetry}

Spectropolarimetry was obtained on April 3.2 and 4.2 by Bjorkman and Nordsieck (1993) at the University of Wisconsin Pine Bluff $0.9 \mathrm{~m}$ telescope. Weighted over the spectral range $3200-7600 \AA$, the results were $0.73 \pm 0.13 \%$ at a position angle of $179.5^{\circ}$ and $0.22 \pm 0.16 \%$ at a position angle of $41^{\circ}$, respectively. The spread in these data is probably due to effects caused by the nearly full moon. Formally combining the two data sets gives $0.46 \pm 0.1 \%$ at a position angle of $5 \pm 6^{\circ}$. Measurements made on April 7.22 UT by Smith (1993) yield a $V$-band polarization of $0.19 \pm 0.11 \%$ at a position angle of $178 \pm 17^{\circ}$. Observations of nearby foreground stars showed less than $0.1 \%$ polarization.

Spectropolarimetry was obtained at McDonald Observatory on April 20 (Trammell, Hines, and Wheeler 1993). The total polarization in the continuum was measured to be $0.9 \%$ at a position angle of $33^{\circ}$, nearly independent of wavelength. The percent polarization decreased at the location of $\mathrm{H} \alpha$ and the position angle rotated by about $10-15^{\circ}$. On April 26, Jannuzi et al. (1993) measured a polarization of $1.04 \%$ at PA = $29^{\circ}$ with similar structure at $\mathrm{H} \alpha$. These results are consistent within the errors. 


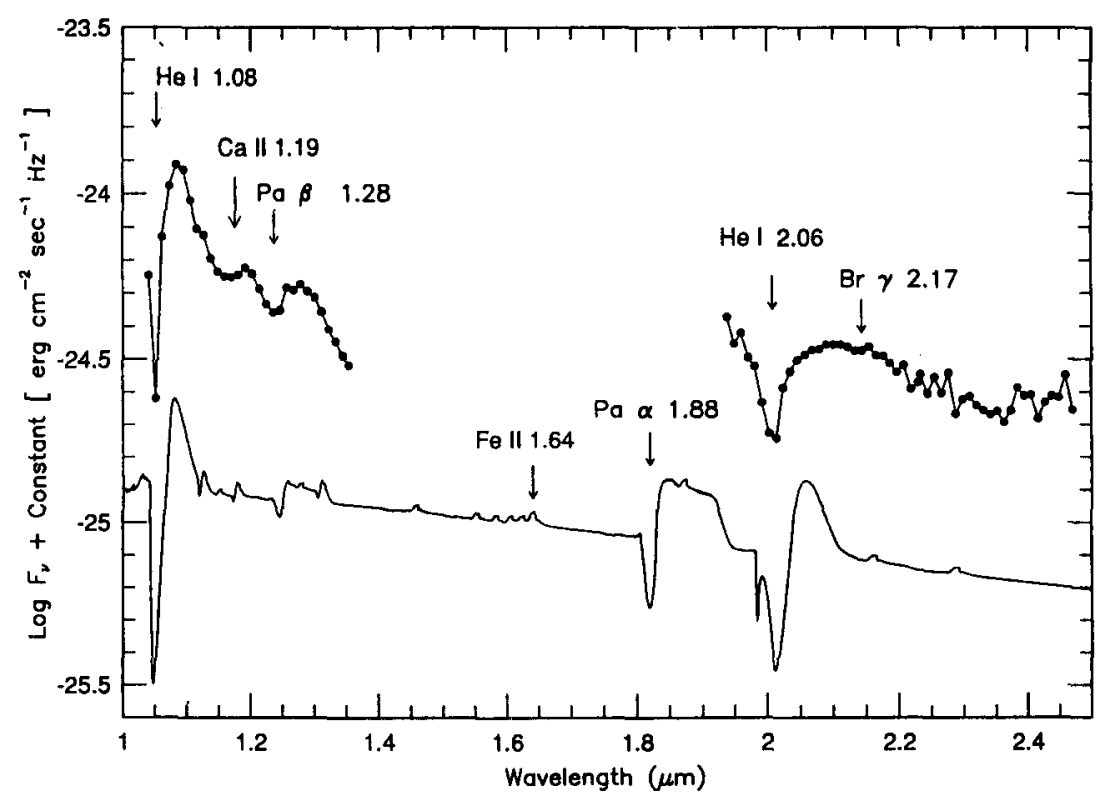

FIgURE 9. Comparison of the May 3.16 infrared spectrum with the spectral model from Fig. 8 (Swartz et al. 1993b; § 13).

At $\mathrm{H} \alpha$ the polarized flux consists of the underlying continuum of the supernova and the flux in the emission line, both affected by any polarization induced by the intervening interstellar medium (ISM). After vector subtraction of the continuum polarization, Trammell et al. found the polarization of $\mathrm{H} \alpha$ emission alone to be $1.1 \pm 0.1 \%$ at a position angle of $150 \pm 4^{\circ}$, similar to the total in the continuum, but with a significantly different position angle. The orientation of this component is compatible with the position angle of the corresponding spiral arm in M81. Trammell et al. assumed this component to be identical to that induced by the ISM in the spiral arm of M81. They then isolated the intrinsic polarization of the supernova by correcting the total polarized flux for the interstellar contribution by means of a Serkowski law. The result for the average continuum polarization of the supernova is then $1.6 \pm 0.1 \%$ at a position angle of $49 \pm$ $3^{\circ}$. The continuum polarization intrinsic to the supernova is found to be significantly larger than the total continuum polarization. The angle differs from that of the total polarized flux and is independent of wavelength. The latter is further evidence that the ISM has been properly isolated and subtracted and that the source of the polarization is electron scattering. The scattered continuum radiation is then isolated by forming the Stokes flux, which does not suffer from the biased error distribution inherent in the polarized flux. The Stokes flux is formed by first rotating all the polarization into a single, rotated Stokes parameter (Trammell, Dinerstein, and Goodrich 1993). This rotated Stokes parameter is then multiplied by the total flux. The intrinsic polarized flux from the supernova computed in this way shows no peak at the rest wavelength of $\mathrm{H} \alpha$, by construction. Data taken on April 30 by Tran and Filippenko (1993) at Lick Observatory with excellent signal to noise ratio confirm this basic interpretation of the spectra. Additional Lick observations are also available (Filippenko 1993b; Miller 1993). There are some differences in the data, but it is difficult to know whether these are due to unequal signal to noise ratios or to an evolution of the polarized flux from the supernova. 


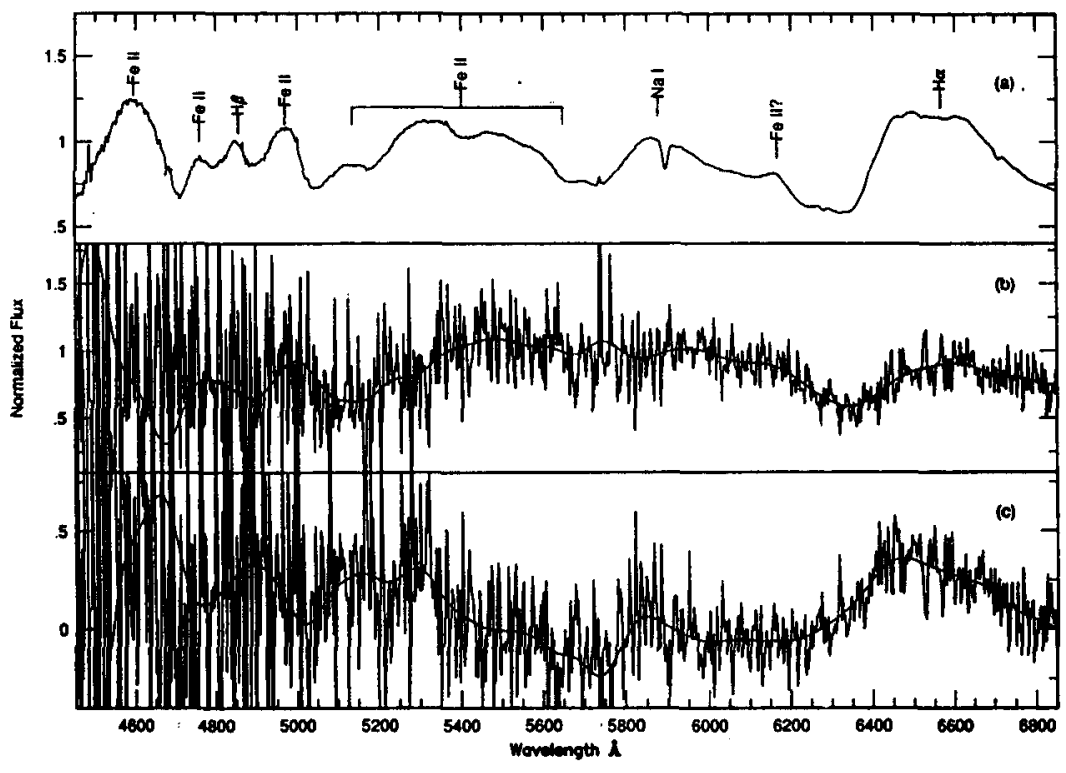

Figure 10. The total flux (a), the Stokes (polarized) flux (b), and the unscattered flux spectra (c) on April 20.11 (Trammell et al. 1993).

In particular, these data suggest a small amount of polarization at $\mathrm{H} \alpha$ intrinsic to the supernova, but it is not clear that this changes the basic interpretation substantially.

Note that if the ISM component has been correctly isolated by Trammell et al. as $1.1 \%$ at a position angle of $150^{\circ}$, then the early measurements of Bjorkman and Nordsieck (1993), and especially the "null" result reported for April 7 by Smith (1993), $0.19 \pm 0.11 \%$ at a position angle of $178 \pm 17^{\circ}$, require special attention. The ISM value cannot change with time. Vector subtraction of the ISM polarization deduced by Trammell et al. from the total polarization of the combined data of Bjorkman and Nordsieck and the total measured in $V$ by Smith yields a polarization for the supernova on April 3-4 of $1.03 \pm 0.1 \%$ at a position angle of $48 \pm 6^{\circ}$ and on April 7 of $1.01 \pm 0.15 \%$ at position angle $55 \pm 4^{\circ}$ (Trammell and Hines 1993). This implies that the supernova already had an intrinsically polarized component on April 7, just after the first minimum. The close similarity of the position angle (and its significant difference from the position angle of any other component) strongly suggests that the intrinsic polarization from the supernova was roughly constant or perhaps slightly increasing from $\sim 1 \%$ on April $3-7$ to $\sim 1.6 \%$ on April 20 at essentially constant position angle, $\sim 50^{\circ}$.

The intrinsic polarized flux from the supernova deduced by Trammell et al. (1993) shows a broad blueshifted $\mathrm{H} \alpha$ absorption, as shown in Figure 10. This feature has a minimum at $\sim 6330 \AA$ corresponding to a velocity of $10,500 \mathrm{~km} \mathrm{~s}^{-1}$ and a FWZI of $\sim$ $395 \AA$ corresponding to a velocity of $18,700 \mathrm{~km} \mathrm{~s}^{-1}$. This absorption feature demands that the flux is already polarized at a depth such that there is still sufficient $\mathrm{H} \alpha$ column depth to induce the absorption feature by scattering the polarized flux out of the line of sight.

The simplest assumption is that the flux from a scattering layer above the photosphere is already polarized as it impinges on the envelope from below. This flux is then absorbed upon passage through the hydrogen layer. This interpretation has the drawback that if the scattering layer is very near the photosphere, the large intrinsic polarization requires 
very large distortions in the core, with axis ratios of order 1.5 to 1 (Shapiro and Sutherland 1982; Trammell et al. 1993). This level of polarization is difficult to generate and maintain in polytropes strongly distorted by differential rotation (Steinmetz and Höflich 1992) or by aspherical explosions in core/envelope configurations or with aspherical winds (Khokhlov 1993).

An alternative possibility is that the flux from the core is unpolarized and that a non-spherical configuration in the envelope creates the polarization through scattering. Rather than a distorted core, this could require a distorted envelope. An advantage of this picture is that with a configuration in which an unpolarized, nearly point source (the core) illuminates a distorted scattering envelope, much less distortion is required to produce the same degree of polarization (Brown and McLean 1977; Fox 1991; Trammell et al. 1993). A distorted envelope is not out of the question given the evidence in many other contexts for bipolar flow associated with late stages of evolution, especially in binary systems. The challenge to this possibility is that the polarization must be created sufficiently deep in the envelope that there remains enough column depth to induce the observed absorption by $\mathrm{H} \alpha$ in the polarized flux. This may be difficult to accomplish in the small $\mathrm{H}$ mass attributed to SN 1993J. We also note that with the understanding that the $\mathrm{H} \alpha$ profile is distorted by He I $\lambda 6678$, there is no direct evidence in the spectrum at this point for any major asymmetries in the envelope.

Another possible interpretation of the polarization data is that both the core and the envelope are basically spherical, but that the interaction with the circumstellar nebula which gives rise to the early narrow lines, the radio emission, and the $\mathrm{X}$-rays $(\S 15)$ is asymmetric, and that the envelope is thus illuminated by $\mathrm{X}$-rays and ionized asymmetrically (Khokhlov 1993).

Although the understanding of the polarization of SN 1993 is at a very rudimentary level, it is clear that the supernova is intrinsically asymmetric in some regard. Whether the polarization is induced in the core, the envelope, or by some interaction with the circumstellar environment, it should evolve in time and in different ways for different models. The spectropolarimetric data will thus be a critical diagnostic for the models. The data of Jannuzi et al. (1993), Tran and Filippenko (1993), and Miller (1993) must be closely examined to check the degree of polarization at $\mathrm{H} \alpha$. If there is remnant polarization at $\mathrm{H} \alpha$ or if, for some reason, there is a minimum in the intrinsic polarization at the position of the unpolarized $\mathrm{H} \alpha$, then the interpretation might be changed.

\section{Models for the Evolution, Dynamics, and Light Curves}

By the time of the meeting a general consensus had emerged about the nature of the progenitor from studies of the light curves. It was accepted that the progenitor had an extended hydrogen envelope of low mass and small hydrogen mass fraction, that the mass of ${ }^{56} \mathrm{Ni}$ ejected was comparable to that of SN 1987A, and that a neutron star remnant was likely. The most common, but not universal, interpretation of the low envelope mass was that it resulted from mass transfer to a binary companion. These models are all variants of the model first presented by Woosley et al. (1987; see also Woosley 1991) for a case which they called a "Type IIb," meaning a model with only a thin hydrogen envelope overlying a helium core which would by itself be a model for a Type Ib supernova. SN 1987K in NGC 4651 was the first known example of this phenomenon (Filippenko 1988). As described above, SN 1993J did develop strong helium lines much like a Type Ib. Many people have thus described SN 1993J as a Type IIb supernova.

Within this framework, most theorists had converged to an even more precisely defined configuration with an initial radius of several hundred solar radii, an ejecta mass of $2-$ 
TABLE 4. Light Curve Model Parameters

(masses in $\mathrm{M}_{\odot} ; \mathrm{R}$ in $10^{13} \mathrm{~cm}$; $\mathrm{L}$ in $10^{38} \mathrm{erg} \mathrm{s}^{-1} ; \mathrm{KE}$ in $10^{51} \mathrm{ergs}$ )

\begin{tabular}{lllllllllll}
\hline \hline Ref. & $\mathrm{M}_{M S}$ & $\mathrm{M}_{e n v}$ & $\mathrm{R}_{e n v}$ & $\mathrm{X}_{e n v}$ & $\mathrm{~L}$ & $\mathrm{M}_{\text {Hecore }}$ & $\mathrm{M}_{N i}$ & $\mathrm{M}_{e j}$ & $\mathrm{KE}$ & mixing \\
\hline 1. & $11-15$ & $.1-.3$ & $2-7$ & $.3-.5$ & $1.5-3.3$ & $2.9-4.5$ & $.06-.08$ & $1.6-4.6$ & $1-1.3$ & some \\
2. & $12-15$ & $.5-.9$ & $2-3$ & 0.2 & 2.7 & $3.3-6$ & $\sim 0.08$ & - & $1-1.2$ & $\mathrm{He}$ \\
3. & - & $<0.2$ & - & - & - & - & 0.05 & - & 1 & - \\
4. & - & 0.75 & 3 & - & - & - & 0.055 & 2.4 & 1.6 & little \\
5. & - & 0.4 & 3 & 0.2 & - & 1.4 & 0.08 & 1.8 & 1 & none \\
6. & 15 & 0.2 & - & - & - & - & 0.15 & - & 0.9 & none \\
7. & $Z 25$ & 3.25 & 7 & 0.5 & $Z 4$ & 9.85 & 0.07 & $\sim 11$ & 2 & He \\
\hline
\end{tabular}

1. Woosley et al. (1994).

2. Shigeyama et al. (1994).

3. Ray et al. (1993).

4. Utrobin (1993).

5. Wheeler et al. (1993).

6. Podsiadlowski et al. (1993).

7. Höflich et al. (1993b).

$3 \mathrm{M}_{\odot}$, and an outer hydrogen envelope of $\lesssim 0.5 \mathrm{M}_{\odot}$ with a hydrogen mass fraction considerably less than $\mathrm{X}=0.5$. Allowing for a neutron star remnant of $\sim 1.5 \mathrm{M}_{\odot}$, the original helium core was $\sim 4 \mathrm{M}_{\odot}$, implying an initial main sequence mass of $\sim 15 \mathrm{M}_{\odot}$. An important exception to this interpretation were the models advocated by Höflich, Langer, and Duschinger (1993b) with main sequence mass in excess of $20 \mathrm{M}_{\odot}$, helium cores of $\gtrsim 6$ $M_{\odot}$, ejecta masses of $\gtrsim 4 M_{\odot}$, and envelopes with more mass and higher hydrogen mass fraction. Various models are summarized in Table 4 . The parameters given are the initial main sequence mass, the envelope mass, the progenitor radius, the $\mathrm{H}$ mass fraction in the envelope, the progenitor luminosity, the mass of the core of helium and heavier elements, the mass of nickel ejected, the kinetic energy at infinity, and a qualitative indication of the degree of mixing of nickel (through He, etc.). Dashes indicate quantities that were either not pertinent to the model or not readily determined from the paper.

In most models the first peak in the light curve is primarily due to the hot, expanding and cooling "fireball" phase that accompanies shock breakout. In this phase, the inner parts expand adiabatically and the outermost, thin regions radiate by diffusion. This fireball phase attends any supernova model, but is faster for smaller radii (e.g., white dwarfs) and generally unobservable. It was observed in SN 1987A. Chevalier (1992) showed that for power law structures with constant opacity, $\mathrm{L} \propto \mathrm{t}^{-x}$ with $x \approx 0.5$. The early luminosity of SN 1993J is also very likely to have been affected by interaction with the circumstellar environment $(\S 14,15)$. Utrobin (1993) proposed that the first maximum is generated entirely by shock heating of an outer shell of matter which might represent the inner layers of a dense wind.

Ray et al. (1993) gave analytic estimates of some of the key parameters. Woosley, Shigeyama, and Utrobin each presented "low mass" numerical light curve models with ejecta masses of $2-3 \mathrm{M}_{\odot}$ and envelope masses of $0.1-0.9 \mathrm{M}_{\odot}$. Similar light curves are described by Podsiadlowski et al. (1993) and Wheeler et al. (1993). As the envelope expands and cools to less than $10,000 \mathrm{~K}$, hydrogen can recombine, decreasing the opacity and allowing the photosphere to recede. For a massive hydrogen envelope, this phase 
corresponds to the normal SN II "plateau" of constant luminosity. In some of the low mass models for SN 1993J, this phase is completely incorporated in the first decline. In other low mass models the recombination phase is postponed because of heating by radioactive decay. In all models, the light curve turns up because of heating by radioactive decay of ${ }^{56} \mathrm{Ni}$ and ${ }^{56} \mathrm{Co}$. The evolution of the location of the photosphere and the resulting implications for the predicted photometric and spectral evolution depend on the model. The light curves are affected by the mass and composition of the ejecta, the opacity, and the nickel mass and distribution. In general, as the ejecta turn optically thin, the light curve falls and attains the slope imposed by the decay of radioactive elements. Although the amount of nickel (as well as a number of other parameters) affects the shape and time of maxima, the principal constraint on the nickel mass as given in these calculations (Table 4) is the comparison to the amplitude of the second peak of the bolometric light curve (e.g., Schmidt et al. 1993; Ray et al. 1993), and hence is proportional to the distance squared.

For models with little or no mixing of the nickel, the photosphere begins to recede shortly after shock breakout and the first minimum is coincident with the photosphere reaching the base of the hydrogen envelope (Woosley et al. 1994; Wheeler et al. 1993). Without a secondary heat input, the light curve would continue to plummet at that point. With little or no nickel mixing, the diffusion wave from this heating arrives at about the time the photosphere recedes through the outer hydrogen envelope and the light curve rises to the second maximum. With no nickel mixing, the underlying helium layer is already rather cool and the opacity is low, so the photosphere tends to recede rapidly through the helium and into the heavy element core (Wheeler et al. 1993). The models of Woosley et al. (1994) invoke some nickel mixing into the helium layers, and this tends to heat and ionize the helium and retard the recession of the photosphere through the helium until the second peak. This may be more consistent with the color evolution, and the onset of the strong He I lines after the second peak, but the latter constrains excessive nickel mixing as discussed in $\S 13$. Shigeyama et al. (1994) found that if the nickel is mixed through the helium layer, hydrogen recombination is delayed due to heating until near the second peak. Höflich et al. (1993b) found similar results for their models, which assumed the nickel to be mixed throughout the helium core. These models also had more massive cores and envelopes, so it is not clear which is the dominant effect. This delayed recession of the hydrogen recombination may not be consistent with the spectral evolution. Most of the low mass models were computed with flux limited diffusion. These models turn optically thin shortly after the second peak. The bolometric luminosity tends to drop too sharply and the colors become completely undetermined. Light curves computed by Podsiadlowski et al. (1993) were representative of the general class of "low mass" models, but omitted any core opacity and hence suffered an extreme version of the core-transparency drop. Woosley et al. (1994) presented the most sophisticated light curves in various colors based on models with R. G. Eastman's time-dependent LTE atmospheres. The atmosphere models smooth out the sudden decrease in luminosity that plagues some of the low mass models when the photosphere reaches the bottom of the metal core. Shigeyama et al. (1994) have managed to avoid the problems associated with the flux-limited diffusion calculations becoming optically thin too precipitously.

Podsiadlowski et al. (1993), Woosley et al. (1994), and Ray et al. (1993) set out the case for a binary companion based on binary evolution computations. They pointed out that binary evolution of moderately massive stars proceeds until the Roche lobe of the mass losing star reaches the inner, helium rich layers left behind by the retreating convective hydrogen-burning core. At this point, the envelope radius drops as further mass is lost, the envelope retreats inside the Roche lobe, and transfer stops. The progenitor 
of SN 1987A might have been the recipient of such a transfer (Tuchman and Wheeler 1989; Rathnasree and Ray 1992; Podsiadlowski et al. 1992). Podsiadlowski et al. (1993) specifically argue that such transfer will leave behind not an $M$, but a $K$ supergiant of about the right color and luminosity to account for the pre-explosion observations of the site. Thus it is quite plausible that prior photometry is revealing the progenitor, but caution should still be exercised in making this identification.

Woosley et al. (1994) argue that the pre-supernova star was filling its Roche lobe at the time of the explosion and thus the hydrogen envelope was highly deformed (about 3:2). This may have implications for the polarization. Woosley et al. (1994) note that any companion star would quickly become embedded in the supernova, but should become visible in three years (perhaps earlier in the $U$ band) when the supernova has faded below $10^{38} \mathrm{erg} \mathrm{s}^{-1}$. Podsiadlowski et al. (1993) and Woosley et al. (1994) point out that if "kicks" have not played an important role, the companion is still bound to the neutron star. For the final separation advocated by Woosley et al. (1994) and Ray et al. (1993), $\sim 10 \mathrm{AU}$, and characteristic ejecta velocities of $\sim 10,000 \mathrm{~km} \mathrm{~s}^{-1}$, the companion would be engulfed in less than about two days, near the first observed peak. It is unlikely that this resulted in any visible display. The entire kinetic energy of the fraction of the envelope impacting on the solid angle of the companion star in the space of a few days only represents about $10^{39} \mathrm{erg} \mathrm{s}^{-1}$. For the bolometric light curve, Woosley et al. (1994) conclude that their lower mass model $\left(11 \mathrm{M}_{\odot}\right.$ initial mass) does the best job with the atmosphere calculation, but the higher mass model $\left(15 \mathrm{M}_{\odot}\right)$ performs better with a fluxlimited diffusion calculation. The color curves are not quite so successful, especially in the blue. The intermediate mass model $\left(13 \mathrm{M}_{\odot}\right)$ does the best job with the colors at the second peak, as shown in Figure 11, but this is also the only model in which hydrogen was mixed into the interior, thus increasing the interior opacity and maintaining the photosphere at larger radii and cooler temperatures for a longer time. In this model, the observed $V$ light curve is too bright on the initial decline and through the first minimum by about 1 magnitude, although the second peak is reproduced fairly well. Woosley et al. (1994) point out that the progenitor envelope can be radiative or convective depending on circumstances, and that this can affect the light curve. Their envelopes are helium and nitrogen rich and carbon poor.

The bolometric light curves in the models of Woosley et al. (1994) never fall on the ${ }^{56}$ Co radioactive decay line. Their model corresponding to an initial mass of $11 \mathrm{M}_{\odot}$ with ${ }^{56} \mathrm{Ni}$ unmixed has a long plateau that may be related to the nickel bubble. The light curve then falls more steeply than the ${ }^{56} \mathrm{Co}$ decay line because of $\gamma$-ray transparency. A similar model with the ${ }^{56} \mathrm{Ni}$ mixed falls off more steeply than the ${ }^{56}$ Co decay after the peak as transparency sets in earlier.

Shigeyama presented work on behalf of his group (see also Nomoto et al. 1993; Shigeyama et al. 1994). They demonstrate that the bolometric light curve of SN 1993J around the second maximum is very similar to that of the Type Ib SN 1983N, and that their helium star models for SN 1983N fit the second bolometric peak of SN 1993J rather well. They conclude that the similarity of SN 1983N and SN 1993J implies that the hydrogen-rich envelope of SN 1993J contains so small a mass that it has relatively little influence on the shape of the second peak. Shigeyama et al. (1994) present models for the bolometric light curve based on helium core masses of $4 \mathrm{M}_{\odot}$ with envelopes of 0.89 and $0.47 \mathrm{M}_{\odot}$ having $\mathrm{Y}$ $=0.80$ and 0.79 , respectively. We note that SN 1993J actually declined somewhat faster than SN 1983N immediately after the second peak (Richmond et al. 1994), although the resemblance is still quite close. Conversely, SN 1983N fell more steeply than SN 1993J on the tail. Nomoto et al. (1993) and Shigeyama et al. (1994) favor mixing of ${ }^{56} \mathrm{Ni}$ to fit the light curves of both SN 1983N and SN 1993J. In the case of SN 1993J, the helium 


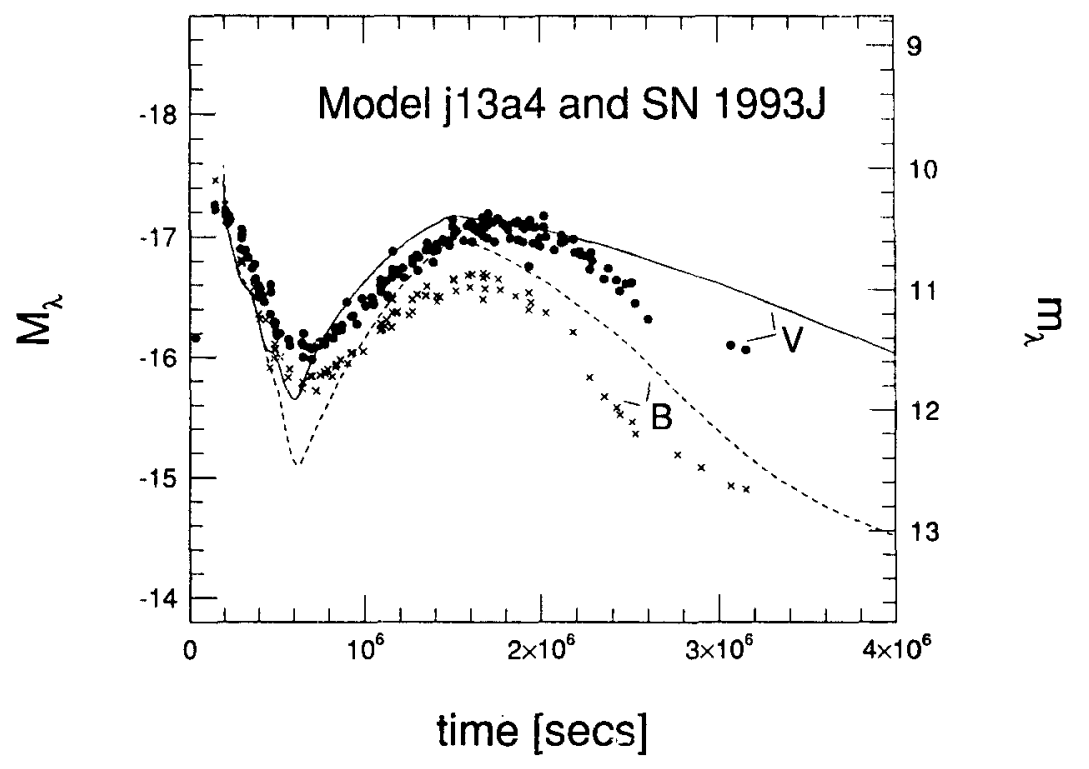

Figure 11. Computed $B$ and $V$ color curves compared with the data for SN 1993J (Woosley et al. 1994).

core expands more slowly due to interaction with the envelope; this gives rise to greater trapping of $\gamma$ rays, thereby yielding a less steep decline on the tail. Neglecting the initial peak which requires an extended envelope, the light curve models of Nomoto et al. (1993) for SN $1983 \mathrm{~N}$ based on bare helium cores with no mixing of ${ }^{56} \mathrm{Ni}$ actually fit the second bolometric peak of SN 1993J better than any of the models of Shigeyama et al. (1994) specifically designed to do so. In the latter, the rise and decline tend to be too steep, and the amplitude with respect to the minimum too great.

Shigeyama et al. (1994) point out that the calculated light curve shape depends on the extent of ${ }^{56} \mathrm{Ni}$ mixing. The time of the light curve minimum is reduced in models with nickel mixing, since it makes heating due to radioactive decays start earlier, as illustrated in Figure 12. They conclude from their models that SN 1993J has actually undergone mixing of ${ }^{56} \mathrm{Ni}$. They also argue that the fact that their calculated luminosities at the second maximum are higher than SN 1993J can be ascribed to excessive expansion velocities at the bottom of the $\mathrm{H}$-rich envelope in the calculation compared with the actual minimum velocity of hydrogen in SN 1993J. They suggest that this can be improved if hydrogen is mixed down into the core to lower velocities, as suggested from the modeling of SN 1987A. Utrobin (1993) argued that the bulk of the radioactive material that powers the second peak is confined to the innermost layers of ejected envelope expanding with velocities less than $\sim 2600 \mathrm{~km} \mathrm{~s}^{-1}$. He concluded that a shell with a positive gradient of hydrogen and a negative gradient of helium must be present and that the hydrogen should be spread throughout the envelope to reproduce the smooth second peak of the visual light curve. The degree of mixing of nickel, hydrogen, and other elements will be constrained by the photometric and spectral evolution. From their attempt to fit the color evolution as well as the bolometric light curve, Woosley et al. (1994) favor a model in which the hydrogen envelope mass is low and the mixing of hydrogen inwards has been small, but in which a small amount of ${ }^{56} \mathrm{Ni}$ has been mixed outwards into the 


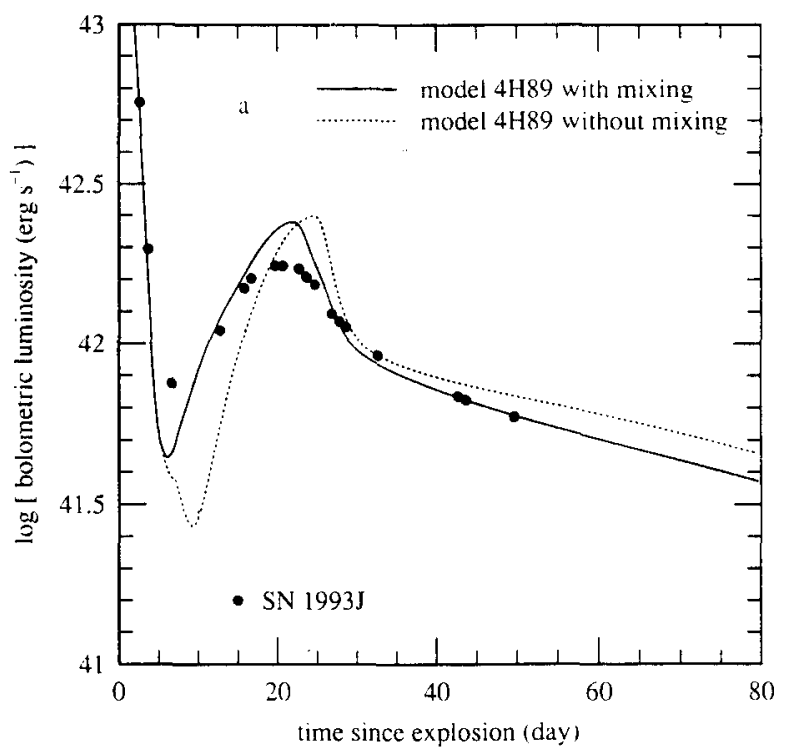

Figure 12. The bolometric data of Schmidt et al. (1993) are shown with two models by Shigeyama et al. (1994), with (solid line) and without (dotted line) mixing of nickel throughout the helium core.

helium core to smooth the light curve near the first minimum. Wheeler et al. (1993) present a model based on the $3.3 \mathrm{M}_{\odot}$ helium core of Shigeyama et al. (1990) with the outer $0.4 \mathrm{M}_{\odot}$ converted to $\mathrm{X}=0.2$. This model has no extended envelope and cannot reproduce the first visual rise. It produces the best $V$ light curve from day 4 to day 30 of any yet presented, and does an adequate job of the bolometric light curve. Since this model confines $\mathrm{H}$ to a distinct layer and includes no ${ }^{56} \mathrm{Ni}$ mixing, it seems premature to come to definite conclusions about the presence of mixing mechanisms. An important additional variable is the opacity. The calculation of Wheeler et al. used "expansion opacities" computed by R. P. Harkness from 42 million lines. Despite the excellent fit to $V$ over some epochs, the model of Wheeler et al. (1993) is clearly inadequate in other ways. It has the typical limitations of flux limited diffusion calculations when the whole core becomes optically thin. This model also gets too blue very suddenly at the first minimum which can probably be attributed to the total omission of any outward mixing of ${ }^{56} \mathrm{Ni}$ so that when the photosphere reaches the inner edge of the hydrogen layer, it recedes rapidly through the helium to the oxygen layer. In any case, care must be taken to avoid misinterpreting the effects of "missing opacity" with mixing of $\mathrm{H}$ or ${ }^{56} \mathrm{Ni}$, both of which will act to increase the local opacity.

Höflich, Langer, and Duschinger (1993b) favored a similar, but quantitatively different model. They computed the progenitor evolution, explosion, $B$ and $V$ band brightness evolution, spectral features, and $\gamma$-ray emission of stellar models in the initial mass range 10 to $30 \mathrm{M}_{\odot}$ at a metallicity of $Z=0.02$ from the zero age main sequence through carbon ignition using an implicit hydrodynamic stellar evolution code (Langer 1991) with OPAL opacities (Iglesias et al. 1992) and mass loss rates according to Nieuwenhuijzen and de Jager (1990). One model had increased rates for the low mass star. The hydrodynamic simulations of the SN explosions were calculated with an explicit PPM hydro-code coupled to an implicit gray radiation transfer program (Höflich et al. 1993a). An extended 
region consistent with the mass loss of the progenitor star was added to the pre-SN structure to study the model SN-wind-interaction. The models are Rayleigh-Taylor unstable in the inner region and they assume that all heavy elements including nickel are mixed throughout the helium core.

Höflich et al. (1993b) concluded that the failure of the low mass models to reproduce the colors at the second peak, as noted by Woosley et al. (1994) and Wheeler et al. (1993), was sufficiently serious to abandon the model. They were also concerned that low mass progenitors $\left(M_{Z A M S} \lesssim 20 \mathrm{M}_{\odot}\right)$, which need binary mass transfer in order to get rid of a large fraction of their envelope, may have problems maintaining a sufficiently strong wind $\left(Z 10^{-5} \mathrm{M}_{\odot} \mathrm{yr}^{-1}\right)$ during their final evolutionary phase to account for the radio and X-ray observations $(\S 14,15)$. They thus advocate a model based on a massive, single star progenitor which has lost the major part of its envelope prior to the explosion. There are several systematic changes as the mass increases. Höflich et al. (1993b) find that the luminosity at the first peak depends to a certain extent on the interaction with the surrounding nebula. Although the luminosity of the first peak is not dominated by this shock interaction as in the suggestion of Utrobin (1993), it amounts to $30 \%$ for their model of initial mass $30 \mathrm{M}_{\odot}, 50 \%$ for $25 \mathrm{M}_{\odot}$, and $60 \%$ for $20 \mathrm{M}_{\odot}$. Larger initial mass also gives more massive envelopes with a smaller He enrichment in the envelope, $\sim 0.5$ versus $Z 0.8$ in some of the lower mass models. As a consequence, the photosphere reaches the $\mathrm{H} / \mathrm{He}$ interface not at the first minimum, as in most of the low mass models, but at about the second maximum. For the high mass models, the second rise is mainly due to the recombination energy from the massive $\mathrm{C} / \mathrm{O}$ core. The result is that the rise to the second maximum is governed by the release of this thermal energy of the helium core as the helium recombination wave proceeds through it. The radioactive decay energy does not become significant until about the second maximum. Since the second peak is affected partially by the rate of recession of the photosphere through the helium in the massive models, the width of the peak is not simply the diffusion time of the radioactive heat from the core. With increasing mass, the overall light curves become brighter because more energy is stored rather than radiated with early recombination. The interaction with a given circumstellar nebula becomes absolutely stronger and the temperatures at the photospheres at the first peak thus increase with mass. To compensate for this effect, a higher reddening is required for a $30 \mathrm{M}_{\odot}$ than for a 20 or $25 \mathrm{M}_{\odot}$ model. Figure 13 shows the $B$ and $V$ light curves for the $30 \mathrm{M}_{\odot}$ model of Höflich et al. (1993b) in which they have assumed $E(B-V)=0.8 \mathrm{mag}$. This model reaches the first maximum on day 5 , when it achieves approximate homology, and the minimum on day 9 . The photospheric temperature is about $55,000 \mathrm{~K}$ at the first peak, and declines to $20,000 \mathrm{~K}$ on day 14 , $12,000 \mathrm{~K}$ on day $22,6000 \mathrm{~K}$ on day 35 , and $4500 \mathrm{~K}$ on day 60 .

There are a number of problems with this high mass model which show that it is extreme and that at least a somewhat lower mass is preferred, as concluded by Höflich et al. (1993b). The major generic problem is that the assumed reddening to make the model work is much too large. It is not even consistent with the model, since $E(B-V)=$ $0.8 \mathrm{mag}$ would imply that the observed color for an infinite temperature black body should be about $0.4 \mathrm{mag}$, and that value of $B-V$ was not observed until about day 10 . The first peak at 5 days is too late. This is a fairly strong constraint, if the time from outbreak to peak of $\sim 2$ days (Wheeler et al. 1993) is accepted. This suggests both the mass and radius of the model envelope are too large. Höflich et al. (1993b) have computed some model spectra and state that they can account for the strong $\mathrm{H}$ lines after the photosphere cools to less that $15,000 \mathrm{~K}$. In this model, however, that only occurs after day 14 and the hydrogen lines became strong around day 5 , prior to the first minimum, 


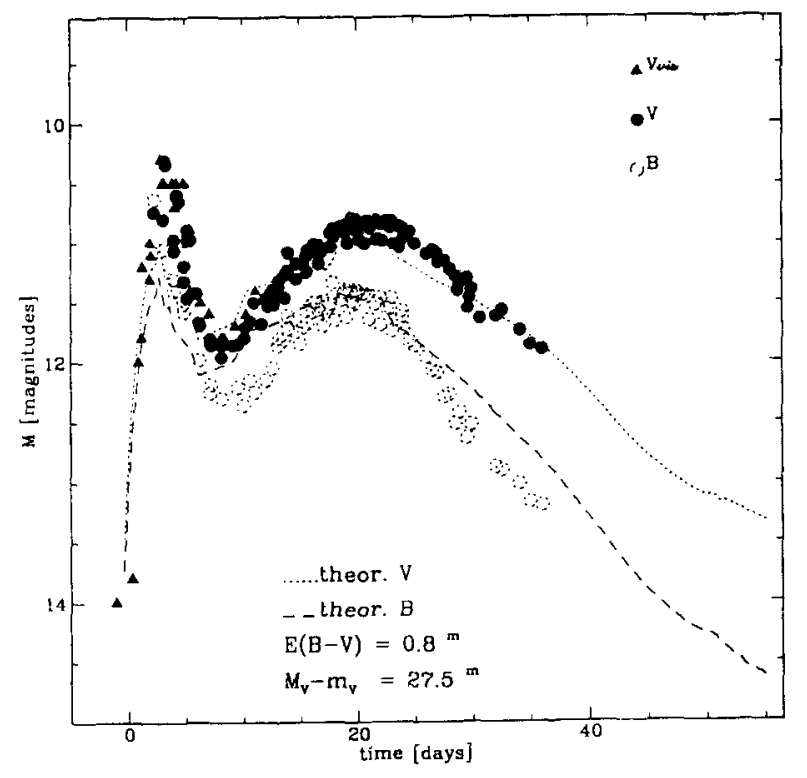

FIGURE 13. Computed $B$ and $V$ color curves compared with the data for SN 1993J (Höflich et al. 1993b).

as shown in Figure 7. This massive model thus seems to be in strong contradiction with the observed spectral evolution.

The major advantage of the high mass model is that it generally provides better fits to both the $B$ and $V$ curves than others proposed. The massive models may also give more opportunity for the hydrogen envelope to both polarize and scatter the flux generated in deeper layers, requiring less geometrical distortion to account for the polarization. The disadvantage is that to fit the photometry, the model requires $E(B-V)=0.8 \mathrm{mag}$, and this is simply disallowed by the accumulating evidence that $E(B-V) \approx 0.15$ mag.

\section{Spectral Modeling}

Although no results were explicitly presented, the evolution of the spectra during the first decline represents a challenge. If the temperature is too low, the hydrogen lines are predicted by atmosphere models to be too strong. This problem has been discussed by Höflich, Baron, Hauschildt, and Harkness (all private communications). Höflich et al. (1993b) advocate a hot atmosphere, but the constraints on the reddening, continuum shape, and colors all argue that the continuum was that of a well-defined cooling black body during the decline. It cannot have been substantially hotter and heavily reddened. Another possibility is that the hydrogen abundance is very low. The smallest $\mathrm{H}$ abundance considered by Höflich et al. was about $40 \%$, as suggested by the more massive evolutionary models. For low mass models, $\mathrm{X} \lesssim 0.2$ is plausible. Baron and Hauschildt have considered such models, but without necessarily resolving the problem. The very early spectra with the weak lines need more consideration.

Baron et al. (1993) presented atmosphere models of optical spectra obtained on April 7.2 and April 13.2. Their synthetic spectra used an approximate lambda iteration method to solve the fully relativistic radiation transport equation and the NLTE rate equations for H I, He I, Mg II, and Ca II (Hauschildt 1992a,b, 1993; Hauschildt and Baron 1993; 


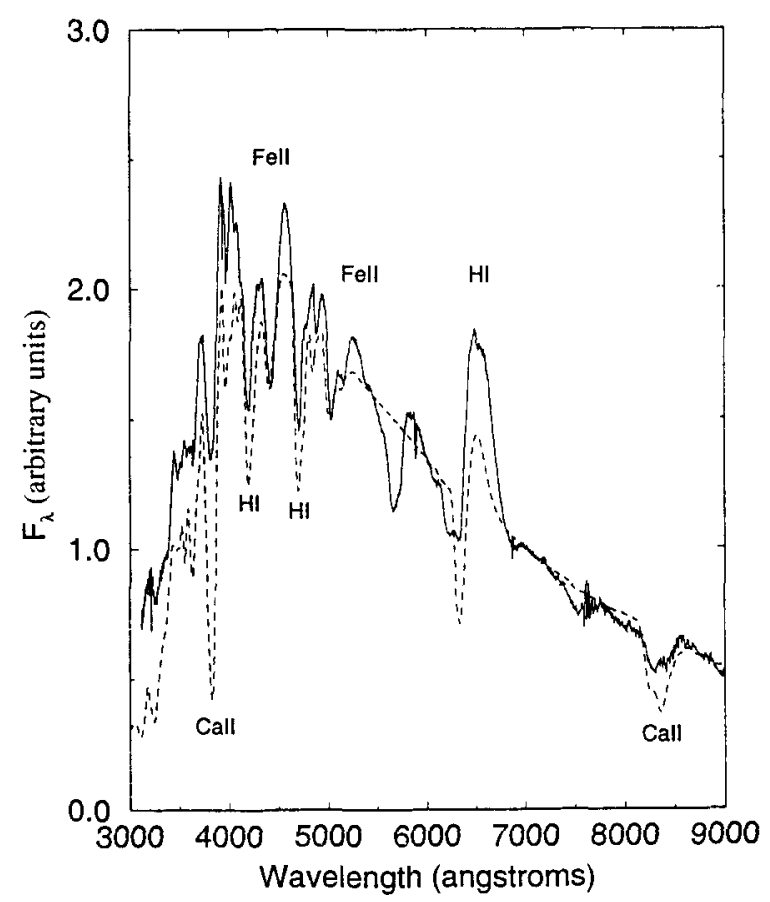

FIgURE 14. A synthetic spectrum (dashed line) is compared to the observed spectrum (solid line) from April 13 (Baron et al. 1993).

Rybicki and Hummer 1991). The line list of Kurucz (1991) was used to select the approximately 200,000 strongest lines with the populations assumed to be in LTE. Model atmospheres were constructed assuming that the velocity profile is homologous, $v \propto r$, and that the density profile follows either a power law, $\rho \propto v^{-N}$, or an exponential falloff, $\rho \propto \exp \left(-v / v_{e}\right)$, where $N$ and $v_{e}$ are parameters to be determined. The radius of the photosphere is given by $R_{0}=v_{0} t$, where the subscript 0 denotes the photosphere. The explosion date was assumed to be Mar 27-28 and the extinction to be $E(B-V)=0.1$ mag. They find that the relatively narrow lines are not due to low photospheric velocity, but rather must be formed in an atmosphere of small extension and hence steep density profile.

On April 7, shortly after the first minimum and after the development of strong $\mathrm{H} \alpha$ emission, Baron et al. (1993) fit the spectrum with $v_{0}=10,500 \mathrm{~km} \mathrm{~s}^{-1}, R_{0}=10^{15}$ $\mathrm{cm}, T_{0}=7,500 \mathrm{~K}$, and $v_{e}=450 \mathrm{~km} \mathrm{~s}^{-1}$. A temperature $T_{0}=8000 \mathrm{~K}$ is allowable, but $T_{0}=7000 \mathrm{~K}$ is too low. Note that Ray et al. (1993) derive a temperature of $6500 \pm 500 \mathrm{~K}$ from the photometry at this time.

Figure 14 displays the April 13 spectrum and a synthetic spectrum with $v_{0}=11,000$ $\mathrm{km} \mathrm{s}^{-1}, R_{0}=1.6 \times 10^{15} \mathrm{~cm}, T_{0}=6,500 \mathrm{~K}$, and $v_{e}=450 \mathrm{~km} \mathrm{~s}^{-1}$. The photospheric velocity has remained nearly constant in time since April 7. This may be more consistent with the low mass models for which the photosphere is in the core during this time 
span than for the high mass models (or those of Shigeyama et al. 1994) for which the photosphere is still receding through the $\mathrm{H}$ envelope, although the absolute value of the photospheric velocity, $\sim 11,000 \mathrm{~km} \mathrm{~s}^{-1}$, is rather high. The decline in photospheric temperature from 7,500 to $6,500 \mathrm{~K}$ is consistent with Ray et al. (1993), within the scatter. Raising the temperature to $T_{0}=7,000 \mathrm{~K}$ results in far too much flux below $4000 \AA$.

The density profiles are extremely steep, with an equivalent power law index $N \gtrsim 20$, especially in comparison with SN $1987 \mathrm{~A}$, which had a power law index $N=4$ at the same epoch (Jeffery and Branch 1990). Baron et al. (1993) were not able to produce the strong emission that is seen in $\mathrm{H} \alpha$. It may be that the nearly pure emission profile of $\mathrm{H} \alpha$ is due to altered level populations produced by radiation from circumstellar interaction or by a flatter density external to the steeply falling profile near the photosphere, as seen in the calculations of Shigeyama et al. (1994). They also fail to fit the broad minimum around $5600 \AA$. Sodium is treated only in LTE in these models, but there is a general feeling that atmospheres at this epoch are too warm for this to be Na I D, so the feature may be He I $\lambda 5876$ (Baron 1993; Eastman 1993; Schmidt 1993). One must then understand the sharp change in this feature when the strong He I lines appear.

Swartz et al. (1993b) have computed atmosphere models at a phase of $\sim 40$ days after shock breakout that account for the development of the strong He I lines in the optical and IR. In the models, the helium lines become visible after (1) the H/He layer recombines, allowing exposure of the helium mantle, (2) the onset of substantial gamma ray deposition in the helium layer, and (3) the free electron density in the mantle has declined sufficiently due to expansion and cooling to eliminate veiling of the helium lines by electron scattering. The structure models employed are the same as in Swartz et al. (1993a), which are helium core models from Shigeyama et al. (1990) but with portions of the helium core turned into hydrogen with variable mass fraction. Note that these models, and the analogous ones used by Wheeler et al. (1993) for light curves, maintain the same density/velocity structure as the original helium core models. They are thus not consistent in this regard with models that include the outer hydrogen envelope $a b$ initio with its associated affect on the dynamics. Likewise, these models could be regarded as the result of some mixing of an outer, more hydrogen-rich layer with part of the helium core. The constraints on hydrogen mixing deduced from these models must be considered in that context.

For a nominal explosion energy of $\sim 10^{51} \mathrm{ergs}$, Swartz et al. (1993b) find that an ejecta mass of $\sim 4.5 \mathrm{M}_{\odot}$ is too large and that of $\sim 1.8 \mathrm{M}_{\odot}$ too low to match the expansion velocities implied by the observed spectra. An ejecta mass of $\sim 2.5 \mathrm{M}_{\odot}$ works well. Of this mass, $\sim 0.4 \mathrm{M}_{\odot}$ contaminated with about $10 \% \mathrm{H}$ by mass gives a good spectral fit, as illustrated in Figure 8. The atmosphere models show that the hydrogen must not be homogeneously mixed with all the helium or the helium lines would be excessively veiled and would not appear in the spectrum. The portion of the ejecta forming the P-Cygni He I lines must therefore be substantially more hydrogen deficient than the $10 \%$ by mass in the outer layer of the model. The hydrogen to helium ratio in the outer layers is not tightly determined. Changing the ratio in the outer $0.4 \mathrm{M}_{\odot}$ from $9 / 1$ to $1 / 9$ only changes the $\mathrm{He}$ I $\lambda 6678$ feature from slightly weaker than $\mathrm{H} \alpha$ to slightly stronger. The latter is observed, so a helium-rich atmosphere is suggested and the observations are consistent with the 1 to 9 ratio assumed in the model illustrated in Figures 8 and 9 . The atmosphere models constrain the nickel mass to be $\lesssim 0.1 \mathrm{M}_{\odot}$ so that the hydrogen is not completely ionized at $\sim 40$ days past shock breakout. Note that this constraint comes from the spectrum at a single epoch and is distance independent. In a similar fashion the nickel cannot be mixed too far out into the ejecta or hydrogen would again be excessively ionized. Homogeneous mixing of the nickel through the helium core seems 


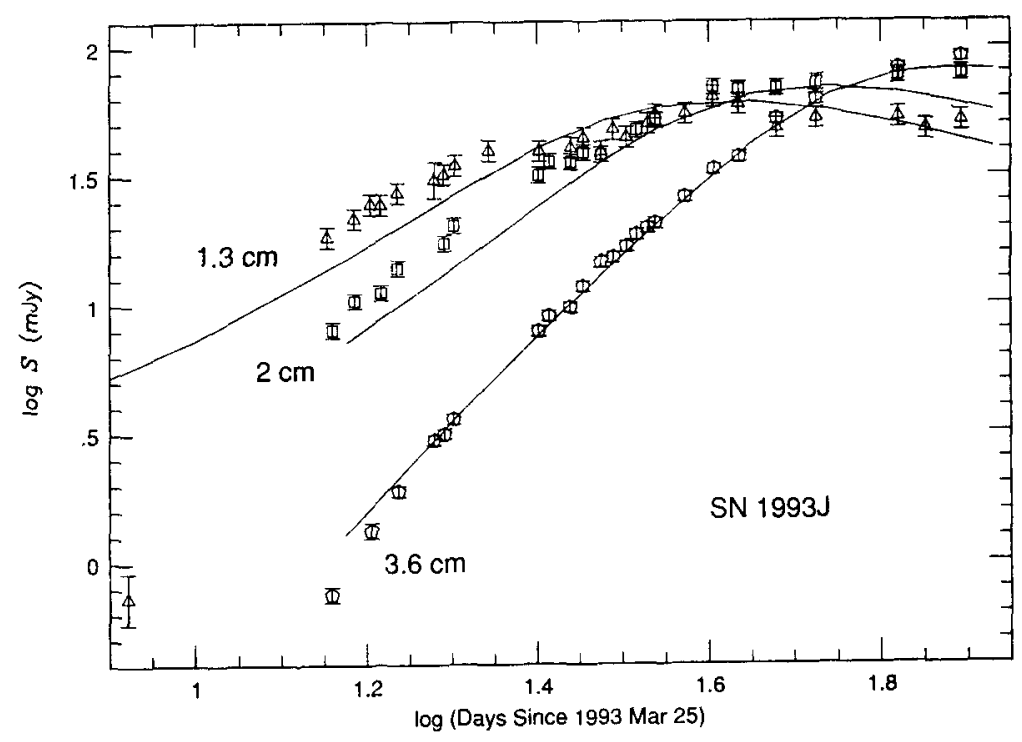

Figure 15. VLA radio observations (Van Dyk et al. 1993b).

to be excessive by this criterion. The models predict that $\mathrm{H}$ and $\mathrm{He}$ should be visible for $\sim 100$ days, but should disappear by $\sim 200$ days. This gives a quantitative basis for the guess by intuition or comparison to other events (SN 1987K; Filippenko 1988) that SN 1993J will resemble a SN Ib in the nebular phase.

\section{Radio}

Van Dyk et al. (1993b) presented observations of the early radio emission made with the Very Large Array at 1.3,2,3.6, 6, and $20 \mathrm{~cm}$, starting on 1993 March 31 UT, less than three days after optical discovery. This radio emission indicates significant interaction of the supernova shock front with circumstellar matter around the progenitor system. The supernova was first detected on April 2 at $0.7 \mathrm{mJy}$ at $1.3 \mathrm{~cm}$. The $1.3 \mathrm{~cm}$ radio light curve has flattened and appears to be turning over as shown in Figure 15. Preliminary indications are that the overall radio properties of SN 1993J are similar to those of several previous Type II radio supernovae (SN 1979C, SN 1980K, and SN 1981K), although SN 1993J was detectable earlier than either SN 1980K or SN 1979C.

D. A. Green (Pooley and Green 1993) reported that radio emission was detected at $15.25 \mathrm{GHz}$, with the Ryle Telescope on Apr 5, only about 8 days after the explosion. As shown in Figure 16, SN 1993J showed an approximately linear increase in flux density from its detection to about $53 \mathrm{mJy}$ at May 3 (day 36) and then a slower rise to a peak of $\sim 80 \mathrm{mJy}$ about day 75 . The $15.25 \mathrm{GHz}$ emission has since shown a general decline, but with significant variations on the time scale of weeks. The linear increase in flux density seen at this frequency lasted several times the delay in detection of the SN. This is difficult to reconcile with the available "mini-shell" models for radio supernovae (Chevalier 1982). Moreover, upper limits on the radio emission for several days before the detection may also be difficult to reconcile with the gradual increase expected from the "mini-shell" model.

Lundqvist, Fransson, and Chevalier (1993) used model 4 H47 of Shigeyama et al. (1994) for the evolution of the photosphere to explore the effects of SN 1993J on the circumstellar 


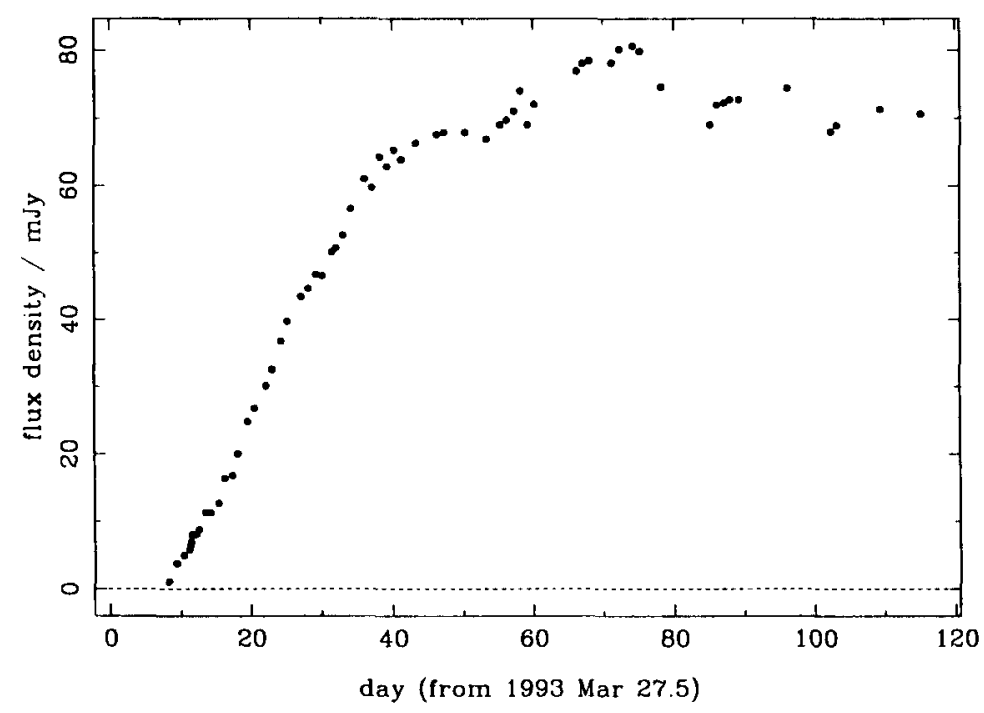

FIGURE 16. 15.25 GHz light curve from Pooley and Green (1993).

medium. The temperature evolution is constrained by IUE observations on March 30.2 and 31.2 when the observed color temperature was $\sim 2 \times 10^{4} \mathrm{~K}$. The peak effective temperature in model $4 \mathrm{H} 47$ is $\sim 2.9 \times 10^{5} \mathrm{~K}$. Lundqvist et al. found that the wind temperature quickly rises to $(1.5-2.3) \times 10^{5} \mathrm{~K}$, and even beyond that very close to the $\mathrm{SN}$. Carbon, nitrogen, and oxygen attain their He-like stages, and $\mathrm{H}$ and $\mathrm{He}$ are fully ionized. After $\sim 0.5$ days, the gas close to the $\mathrm{SN}$ starts to recombine. With a mass loss rate of $2 \times 10^{-5} \mathrm{M}_{\odot} \mathrm{yr}^{-1}$ and a wind speed of $10 \mathrm{~km} \mathrm{~s}^{-1}$, the gas close to the SN cools down to $\sim 2 \times 10^{4} \mathrm{~K}$ in $\sim 0.5$ days, whereas outside $4 \times 10^{15} \mathrm{~cm}$ it is still $2 \times 10^{5} \mathrm{~K}$. During the recombination period that follows, the observed $\mathrm{H} \alpha$ and $\mathrm{N} \mathrm{V} \lambda 1240$ emission is likely to form. Collisional excitation alone is not sufficient to power the $\mathrm{N} \mathrm{V}$ line. Photoexcitation is also needed; however, the photospheric continuum flux at $1240 \AA$ in the models of Shigeyama et al. drops too fast during the first day, giving an order of magnitude too low a N V $\lambda 1240$ flux when the line was observed, i.e., after $\sim 2-3$ days. It is therefore likely that the $\mathrm{N} V$ line was powered by a somewhat harder photospheric flux after $\sim 0.5$ days than in model $4 \mathrm{H} 47$, and/or by the radiation from the interaction region. The $\mathrm{H} \alpha$ line is due to recombination emission, and indicates a high wind density corresponding to a mass loss rate of at least $5 \times 10^{-5}\left(w / 10 \mathrm{~km} \mathrm{~s}^{-1}\right) \mathrm{M}_{\odot} \mathrm{yr}^{-1}$, where $w$ is the wind speed. For a constant speed of $w=10 \mathrm{~km} \mathrm{~s}^{-1}$, Van Dyk et al. (1993b) estimated the mass loss rate from the progenitor to be $\dot{M} \approx 4 \times 10^{-6} M_{\odot} \mathrm{yr}^{-1}$ using an assumed unshocked wind temperature $T_{w}=10^{4} \mathrm{~K}$ and a shock velocity of $10,000 \mathrm{~km}$ $\mathrm{s}^{-1}\left(\dot{M} \propto T_{w}^{0.68} v_{\text {shock }}^{1.5}\right)$. Lundqvist et al. (1993) noted that the observed slow turn-on is not well modeled by a simple, isothermal, $r^{-2}$ wind, but indicates that $\dot{M}$ is close to $4 \times 10^{-5}\left(w / 10 \mathrm{~km} \mathrm{~s}^{-1}\right)\left(T_{w} / 10^{5} \mathrm{~K}\right)^{3 / 4} \mathrm{M}_{\odot} \mathrm{yr}^{-1}$. With their estimate that $T_{w}$ is of order $10^{5} \mathrm{~K}$ due to the pre-ionization by the shock breakout, they concluded that $\dot{M} / w$ is about an order of magnitude higher than estimated by Van Dyk et al.

Lundqvist et al. (1993) explored ways of obtaining a slow radio turn-on. A density profile of $\rho \propto r^{-1.5}$, implying a departure from a steady state, agrees with the data. Other ways to produce a slow turn-on are for the wind to be blobby, for the wind temperature close to the shock to decrease with time while the radio optical depth is close to unity, 
and for the wind to have an asymmetrical density distribution. Since the slow turn-on is observed at many wavelengths and thus over an extended period of time, it appears as if a decreasing wind temperature is not likely. Van Dyk et al. (1993b) argue that the relatively slow radio turn-on may indicate the presence of internal absorption which is generally seen only in supernovae with more massive progenitors. They suggest that such internal absorption may be evidence for the presence of filamentary or "blob" structures or non-spherical distortions in the radio generation region (Weiler et al. 1990). Lundqvist et al. (1993) noted that asymmetry and blobs may also be helpful in explaining the high wind density needed for the observed high $\mathrm{H} \alpha$ flux. Asymmetry in the radio generation region could be consistent with the binary models presented at the meeting.

\section{X Rays}

B. Aschenbach reported that on April 3, six days after the optical detection, soft Xray emission from SN 1993J was detected by the ROSAT observatory during a 2700 sec exposure (Zimmermann et al. 1993a). This marks the earliest detection of X-rays after a supernova explosion. A preliminary analysis of the data shows that the mean spectrum in the ROSAT energy range between 0.1 and $2.4 \mathrm{keV}$ can be fit by either a power law with photon index $-0.88 \pm 0.13$ or by thermal models with $k T$ well above $6 \mathrm{keV}$ (Zimmermann et al. 1993b). With a distance of $3.5 \mathrm{Mpc}$ to M81 an X-ray luminosity of $1.7 \times 10^{39} \mathrm{erg} \mathrm{s}^{-1}$ in the $0.1-2.4 \mathrm{keV}$ band is calculated. The ROSAT position coincides within $5^{\prime \prime}$ to the optical position. H. Tsunemi reported preliminary results for the ASCA team (Tanaka et al. 1993). ASCA first detected X-rays from SN 1993J on April 7 in the energy range $0.5-10 \mathrm{keV}$. Observations were repeated several times until May 18 when they had to be halted due to the solar angle constraint. The maximum luminosity is about $5 \times 10^{39}$ $\mathrm{erg} \mathrm{s}^{-1}$. The X-ray intensity decayed monotonically with an e-folding time about 50-70 days. A linear fit to the SN 1993J flux within the first month shows a decrease of about $30 \%$ in 10 days, inconsistent with a constant count rate at a confidence level of more than $4 \sigma$ (Zimmermann et al. 1993c). It is also clear that the supernova flux did not undergo larger changes over the time scale of one month. The hydrogen column density of $5 \times 10^{20}$ $\mathrm{cm}^{-2}$ does not require large amounts of intrinsic absorption above the Galactic value of $4.3 \times 10^{20} \mathrm{~cm}^{-2}$ in the direction to M81. This is consistent with the assumption that the strong radiation from the supernova effectively ionized the circumstellar medium in front of the emission region.

Theories for the early X-ray emission must account for the high temperatures in the emission region, a very low intrinsic absorption, the fact that $\mathrm{X}$-rays were detected very early after the explosion when radio emission was just starting to rise, as well as for the relatively constant behavior of the soft $\mathrm{X}$-ray luminosity over a time scale of one month. In many models the early soft X-ray flux is expected to decrease over time scales of a few weeks or months, depending on the density profile of the circumstellar material near to the collapsed object. The present observations do not support these expectations, which were derived using simple assumptions on the mass loss rate and the wind velocities of the red supergiant progenitor.

Lundqvist et al. (1993) noted that the observed X-ray spectrum is hard $\left(k T_{X}>10\right.$ $\mathrm{keV}$ ) and flat, and there is only little material absorbing the X-rays. A lower column density of the gas absorbing the X-rays means that the circumstellar medium should either have a low density or should be nearly fully ionized. In addition, the flux in the observed band decays slowly, roughly as $t^{-0.3}$. The $\mathrm{X}$-rays are likely to be produced behind the reverse shock going into the supernova ejecta. The expected spectrum is very different depending on whether or not the shock is radiative. It is flat if the shock is 
adiabatic, but if radiative, the radiation is expected to be blocked out almost entirely by a cool shell next to the contact discontinuity between the shocked ejecta and the shocked circumstellar gas (e.g., Chevalier and Fransson 1994). Because of the low column density of absorbing matter, the shock is therefore most likely adiabatic. Assuming a free-free spectrum, the X-ray luminosity per energy unit in the observed band is expected to decay roughly as $t^{-(n-4) /(n-2)}$, where $n$ is a power-law index describing the density structure of the not yet reverse-shocked ejecta, $\rho \propto r^{-n}$. The decay rate may be different if electrons and ions are not in full energy equipartition. From the facts that $k T_{X}>10 \mathrm{keV}$ and that the ratio of the cooling time scale to the dynamical time scale increases with decreasing $n$, Lundqvist et al. find that $n<10$. Best fits to the luminosity in the ROSAT and ASCA bands indicate $n \approx 7$ and a mass loss rate of $\dot{M} \approx(2-3) \times 10^{-5}\left(w / 10 \mathrm{~km} \mathrm{~s}^{-1}\right)$ $\mathrm{M}_{\odot} \mathrm{yr}^{-1}$.

It is not entirely ruled out that the $\mathrm{X}$-rays may come from the forward shock moving into the circumstellar medium, though Lundqvist et al. (1993) find it less likely. In early phases, this radiation is dominated by inverse Compton scattering of the photospheric radiation field (e.g., Fransson 1984; Lundqvist and Fransson 1988), and the time when the shock turns adiabatic depends strongly on whether electrons and ions are in full equipartition. For typical parameters one obtains an order of magnitude lower temperature of the electrons $\left(T_{e}\right)$ compared to the ions $\left(T_{i}\right)$ if the electrons are heated only by Coulomb collisions. The Compton scattering creates a high-energy power-law tail to the photospheric spectrum. The power-law index is $\sim 1$ if $T_{e}=T_{i}$, and $>2$ if $T_{e}<T_{i}$. Because the observed spectrum was flat, the Compton tail is unlikely to have been seen. This may favor a non-equipartition situation.

Suzuki et al. (1993) use the hardness ratio $A / R=L_{A} / L_{R}$ between the ASCA $1-10 \mathrm{keV}$ luminosity $\left(L_{A}\right)$ and the ROSAT $0.1-2.4 \mathrm{keV}$ luminosity $\left(L_{R}\right)$ to compare models and the observations. Around the dates of the ASCA and ROSAT observations, $L_{A} \approx 5 \times 10^{39}$ erg $\mathrm{s}^{-1}$ and $L_{R} \approx 1.6 \times 10^{39} \mathrm{erg} \mathrm{s}^{-1}$, so that $A / R \approx 3$. Suzuki et al. modeled the collisions between the supernova ejecta and the circumstellar matter with a series of models with two parameters, the expansion velocity at the outer edge of the ejecta and the power law index in the density distribution $-n=d \ln \rho / d \ln r$ in the outermost layers. The initial density distribution of the circumstellar matter is assumed to be $n=1 \times 10^{9}\left(r / 1 \times 10^{14} \mathrm{~cm}\right)^{-2}$ amu $\mathrm{cm}^{-3}$. This density distribution is required to reproduce the observed $\mathrm{X}$-ray luminosities, hardness, and relatively slow decline rate. It is higher than that of Van Dyk et al. (1993b), but consistent with that of Lundqvist et al. (1993).

To reproduce the observed hardness of the X-ray emission, the expansion velocity at the outer edge of the ejecta at the beginning of the collision must be as high as $\sim 5 \times 10^{4}$ $\mathrm{km} \mathrm{s}^{-1}$. This can be consistent with models of low envelope mass (Nomoto et al. 1993). In addition, the temperature in the shocked ejecta should be as high as $(2-3) \times 10^{8}$ $\mathrm{K}$ for a fairly long time. This suggests that the ejecta have a relatively shallow density gradient $(\mathrm{n} \approx 8)$. As shown in Figure 17 , Suzuki et al. (1993) predict the decline of the X-ray luminosity, softening of the spectra, and growing line features which should be fairly broad due to rapid expansion of the shocked ejecta. Shigeyama et al. (1994) predict that the hard X-rays from a pulsar can be observed with ASCA in about three years if the pulsar luminosity is as high as that of the Crab Nebula.

\section{Gamma Rays}

With an optimal model for hard emission, Woosley et al. (1994) predict a flux of $\lesssim 2 \times 10^{-5}(2.5 \mathrm{Mpc} / D)^{2}$ photons $\mathrm{cm}^{-2} \mathrm{~s}^{-1}$ in the $847 \mathrm{keV}$ line of ${ }^{56} \mathrm{Co}$ at peak during 


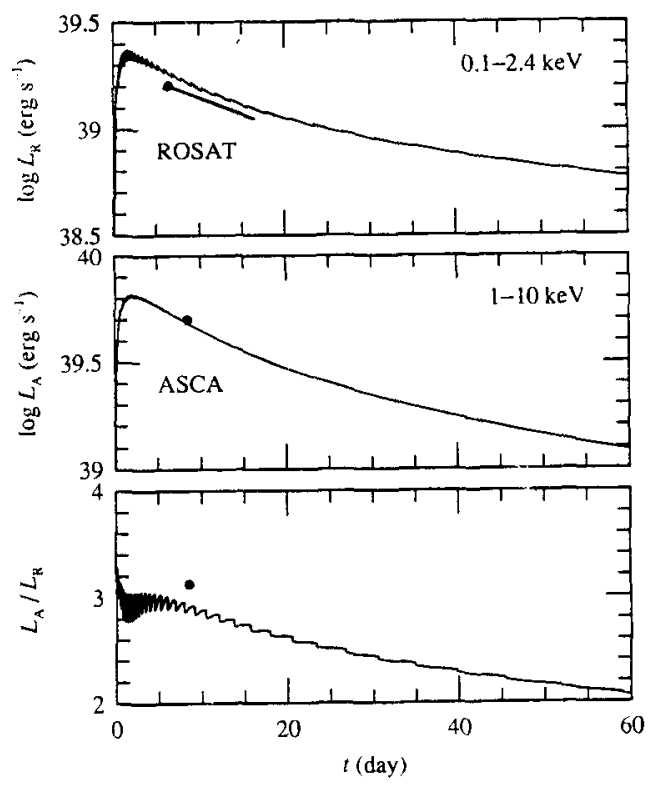

FIGURE 17. ROSAT and ASCA X-ray observations shown in comparison with models of Suzuki et al. (1993).

August 1993, and a Comptonized continuum which peaks at a few $\times 10^{-4}$ photons s ${ }^{-1}$ $\mathrm{cm}^{-2} \mathrm{MeV}^{-1}$ at $40 \mathrm{keV}$ a few months after the explosion. Nomoto et al. (1993) and Shigeyama et al. (1994) note that if substantial mixing of ${ }^{56} \mathrm{Ni}$ occurred and the mass of ${ }^{56} \mathrm{Ni}$ is as large as $\sim 0.15 \mathrm{M}_{\odot}$, the $\gamma$-ray lines (and hard X-rays) from $\mathrm{SN} 1993 \mathrm{~J}$ may be marginally observed with CGRO at maximum around day 130 . The distance is actually closer to $3.5 \mathrm{Mpc}$, most models suggest a nickel mass of $<0.1 \mathrm{M}_{\odot}$, and constraints from the spectral modeling of the helium lines suggest that outward mixing of the nickel is small, so these predictions must be regarded as optimistic.

SN 1993J was observed with OSSE on CGRO during three different intervals, approximately 9-15, 23-36, and 93-121 days after outburst (Chipman 1993). There may be continuum emission below $200 \mathrm{keV}$ in the intervals prior to and near the second optical peak with intensities at $100 \mathrm{keV}$ of $(1.82 \pm 0.39) \times 10^{-3}$ photons $\mathrm{cm}^{-2} \mathrm{~s}^{-1} \mathrm{MeV}^{-1}$ and $(0.89 \pm 0.35) \times 10^{-3}$ photons $\mathrm{cm}^{-2} \mathrm{~s}^{-1} \mathrm{MeV}^{-1}$, and photon indices of $-2.3 \pm 0.5$ and $-2.2 \pm 0.9$, respectively. Although this emission cannot be unambiguously attributed to SN 1993J (because of the large OSSE field of view), it is worth noting that OSSE did not observe flux at this level in observations 597 and 443 days prior to SN 1993J. A power law of fixed photon index -1.2 approximately extrapolates from the first OSSE observation to the total luminosity in the range 1-10 keV measured by ASCA one day earlier (Tanaka et al. 1993). The flux at $200 \mathrm{keV}$ is probably too bright to be entirely due to Comptonized $\gamma$-rays from radioactive ${ }^{56} \mathrm{Ni}$ and ${ }^{56} \mathrm{Co}$. There was no evidence for line emission in any observation and no evidence for emission of any kind in the longer, more sensitive, third observation when $\gamma$-ray lines were most likely to be detectable. Preliminary measured fluxes of the $847 \mathrm{keV}$ and $1238 \mathrm{keV}$ lines are $(1.6 \pm 2.1) \times 10^{-5}$ photons $\mathrm{cm}^{-2} \mathrm{~s}^{-1}$ and $(0.7 \pm 1.9) \times 10^{-5}$ photons $\mathrm{cm}^{-2} \mathrm{~s}^{-1}$, respectively.

Höflich et al. (1993b) noted that the high mass models tended to predict less $\gamma$-ray 
flux than the lower mass models, but since even very optimistic low mass models did not predict a flux above the $1 \sigma$ limits reached by CGRO, the mass cannot be constrained by this non-detection.

\section{Distance Estimates}

A number of recent distance estimates to M81 by different methods including the planetary nebula luminosity function (Jacoby et al. 1989) and the surface brightness fluctuations (Tonry 1991) give a distance $D=3.47 \pm 0.17 \mathrm{Mpc}$ and distance modulus DM $=27.7 \pm 0.1 \mathrm{mag}$ (de Vaucouleurs 1993). A new determination of Cepheid distances with the Hubble Space Telescope gives $\mathrm{DM}=27.8 \pm 0.2 \mathrm{mag}$ corresponding to $D=3.63 \pm 0.34$ Mpc (Freedman et al. 1994). There are also a variety of ways to use SN 1993J to estimate the distance.

From simultaneous least squares fits to the optical continua, Wheeler et al. (1993) determine $\mathrm{T}=14,900 \pm 1000 \mathrm{~K}$ on March 31.18 and $\mathrm{T}=11,800 \pm 700 \mathrm{~K}$ on April 1.14. Adding several other high quality spectra does not seem to change these estimates appreciably. In the black body limit, assuming no significant change in photospheric velocity between March 31 and April 1, Wheeler et al. find $D=4.2 \pm 0.6 \mathrm{Mpc}\left(v / 10^{4}\right.$ $\mathrm{km} \mathrm{s}^{-1}$ ), or DM $=28.1 \pm 0.4 \mathrm{mag}$ for $v=10^{4} \mathrm{~km} \mathrm{~s}^{-1}$. The velocities are not well determined from the weak lines at such an early epoch, but are about that value or perhaps $10 \%$ higher. Effects of scattering and geometrical extension may decrease this estimate, although it seems fairly independent of the assumed reddening. The empirical basis for the continuum temperatures appears fairly well established, but the geometry is not well known. If the effective density distribution is very steep because of interaction with the circumstellar matter, then geometrical and scattering effects could be very small.

De Vaucouleurs (1993) has used $U-B$ and $B-V$ colors during the first week after shock breakout to determine the reddening and temperature independently. For $E(B-V)=$ $0.15 \mathrm{mag}$ and $E(U-B)=0.11 \mathrm{mag}$ (of which two-thirds is Galactic foreground), he derives $\mathrm{T}=14,800$ for March 31.2 and 11,400 for April 1.2. Combining the temperature as a function of time, and the radius $R=R_{0}+v\left(t-t_{0}\right)$ derived from expansion velocities measured at Lick Observatory and at McDonald Observatory, he obtains a mean distance modulus of $27.7 \pm 0.1 \mathrm{mag}$ which is nearly independent of initial conditions. The method begins to fail after day 6 when departures from a Planck curve become significant.

Baron et al. (1993) obtain photospheric temperatures, velocities, and radii by adjusting the parameters of their atmosphere models to match the observed continua and lines. They get velocities of about $11,000 \mathrm{~km} \mathrm{~s}^{-1}$ on both April 7 and April 13. By comparing synthetic photometry from these models to observations they estimate the distance modulus to be $\mathrm{DM}=28.0 \pm 0.3 \mathrm{mag}(D=4.0 \pm 0.5 \mathrm{Mpc})$ and an explosion date of 1993 March $27.5 \pm 0.5 \mathrm{UT}$, assuming $E(B-V)=0.1 \mathrm{mag}$. They caution that their distance estimate is dependent on the assumed extinction, the assumption of spherical symmetry, and whatever changes are required to fit the $\mathrm{H} \alpha$ and $5600 \AA$ features.

Schmidt et al. (1993) use the black body corrections as a function of color temperature computed from a grid of Type II models, their own $B V I$ photometry for April, and velocities from spectra to estimate distances. They employ the weak metal lines such as Fe II $\lambda 5169$ and $\lambda 5018$ to estimate the velocity of the photosphere and find that it dropped from $7900 \mathrm{~km} \mathrm{~s}^{-1}$ on 15.1 April, just before the second peak, to $6600 \mathrm{~km} \mathrm{~s}^{-1}$ on 24.1 April, about a week after the peak, to $5400 \mathrm{~km} \mathrm{~s}^{-1}$ on 17.5 May, about day 50 when the light curve is on the tail. They use these data to determine a distance of $2.6 \pm 0.4$ $\mathrm{Mpc}$ and the time of explosion to be March $26 \pm 3$. They note that the models adopted 
to estimate the black body correction may not apply to events like SN 1993J which have lost a great deal of mass, are colliding with the lost mass, and are asymmetric.

The discrepancy between Baron et al. (1993) and Schmidt et al. (1993) seems to arise primarily from the different photospheric velocities that they deduce, $11,000 \mathrm{~km} \mathrm{~s}^{-1}$ on April 13 for Baron et al. and $7900 \mathrm{~km} \mathrm{~s}^{-1}$ on April 15 for Schmidt et al. (Schmidt 1993). Other things being equal, a slower photosphere will give a proportionately smaller distance, and the two distance estimates seem to differ by about the ratio of these velocities. Resolution of this discrepancy should give more insight into the nature of the atmosphere of SN $1993 J$.

\section{Discussion}

While its nearby location and relative brightness ensured that it would be very well studied, SN 1993J continues the tradition of SN 1987A by being peculiar and very much worthy of study in its own right.

The light curve and spectral modeling done by the time of the Xian conference point rather strongly to a picture in which a star of initial mass $\sim 15 \mathrm{M}_{\odot}$ transferred most of its hydrogen envelope to a companion, then exploded by core collapse to form a neutron star. The supernova has only $2-3 \mathrm{M}_{\odot}$ of ejecta, including a small amount of helium enriched, hydrogen envelope and $\sim 0.05-0.1 \mathrm{M}_{\odot}$ of ${ }^{56} \mathrm{Ni}$. This picture is suggested by the following:

- The pre-discovery photometry that is consistent with an early $\mathrm{K}$ supergiant progenitor which is, in turn, consistent with extensive mass loss to a binary companion that is shut off when the envelope shrinks in mass to expose helium-rich layers. - The initial peak about two days after shock outbreak that is reproduced by models with low mass envelopes of low $\mathrm{H}$ abundance and initial radii of several hundred solar radii.

- The reproduction of the rise of the bolometric flux from the first minimum to a second maximum in about two weeks and then the subsequent decline onto a tail.

- The radio, UV, and X-ray observations that show the interaction with a dense circumstellar medium that is probably not distributed like a simple steady-state wind.

- Atmosphere models that reproduce the epoch and spectral characteristics of the onset of strong He I lines.

A number of areas need more work to confirm or deny this general model. It is not clear that the progenitor has been properly identified. There are at least two stars within the 17 parsec seeing disk around the supernova, but it is not certain that either is the progenitor. Photometry of the progenitor may not be resolved until the supernova fades. The current photometry can certainly set a color and model dependent upper limit to the mass of the progenitor. A single very massive progenitor $\left(M \gtrsim 20 \mathrm{M}_{\odot}\right)$ is unlikely because the measured luminosity of the composite of stars at the position of SN 1993J is too low. The progenitor evolution requires more study. The mass transfer from the red supergiant progenitor is expected to occur on dynamical time scales until the mass ratio is reversed. This causes the companion to expand and leads to a common envelope. There have been no calculations of the response of the mass receiving companion to such a rapid mass accretion. Many of the low mass models that reproduce the initial peak reasonably well have not included any luminosity from the interaction with the circumstellar nebula, nor any effects of the non-homologous expansion that will be induced. None of the light curve models have addressed the effects of ionization from the X-rays produced by the interaction with the circumstellar matter. None of the models have yet been able to reproduce the color evolution properly.

The high mass models presented so far do a better job than most low mass models in 
reproducing the color evolution, but require an excessive reddening and do not seem to be in accord with the observed spectral evolution. The high and low mass models can be tested by continued comparison to the light curve and spectral evolution. The high mass models predict a different evolution of the photosphere and so will predict different general properties for the evolution of the helium lines, the onset and nature of the nebular spectrum, and the evolution of the spectropolarimetry. Similar arguments can be made about the degree of mixing of the nickel outward, mixing within the core, and mixing of the core with the envelope. Hydrodynamic models give an a priori expectation of mixing, but excessive mixing of nickel outward and hydrogen inward seem discouraged by the spectral constraints, especially the epoch of onset of the strong He I lines. The spectral models can only constrain microscopic mixing of $\mathrm{H}$ and $\mathrm{He}$, and so macroscopic mixing of "blobs" may be consistent with the observations. The constraints on the mixing of nickel are more general because of the diffuse effect of the associated gamma rays. It is important to note that the spectrophotometry can constrain the amount and degree of mixing of the nickel even though nickel and its decay products are not observed directly in the spectrum.

Much remains to be done to understand the nature of the circumstellar medium. Given the evidence that the density profile does not simply scale as $\rho \propto r^{-2}$, the surrounding nebula is not simply the product of a spherical steady-state wind. It may thus be very deceptive to speak of an associated "mass loss rate" in the sense that the loss rate is derived by making the steady state assumption. Given the likelihood of binary interaction, we are probably looking at some complex combination of flows and common envelope. While it may be true that stars with initial mass $\sim 15 \mathrm{M}_{\odot}$ are unlikely to drive a wind with the density implied by the radio, UV, and X-ray observations, it is not clear that one must rely on a simple wind to provide the density.

Much more work must be done on the early light curve and spectral modeling to incorporate the effects of the collision with the circumstellar matter. Outward and reverse shocks and the hard radiation from them will affect the density and temperature distribution of the outer ejecta. The evolution near the first peak and even later is unlikely to be homologous. The models with envelopes of $\$ 0.2 \mathrm{M}_{\odot}$ become homologous in a day or so, but increasing the mass of the envelope or including the circumstellar matter will serve to delay the approach to homologous expansion. The spectra on the first decline are a particular challenge. The features have very small amplitude even though the temperatures are moderately low and simple models predict stronger lines. The expected low $\mathrm{H}$ abundance may play a role and the hydrogen may be excessively ionized by the shock-produced X-rays. The X-rays must not, however, heat the photosphere excessively since the observations show $\mathrm{T} \lesssim 15,000 \mathrm{~K}$ on the first peak.

The $\mathrm{H}$ lines and other features begin to get distinct shortly before the first minimum. This will set important constraints on the distribution of the density and composition, including the nickel. The latter affects the location of the photosphere and hence the column depth contributing to the strength of the lines.

SN 1993J has been reasonably well studied in the optical, but it is not clear that coverage in the IR has been adequate. Further IR observations are encouraged to follow the evolution of the $\mathrm{H}$ and $\mathrm{He}$ lines, to look for evidence of dust and molecule formation, and perhaps even to detect the IR lines of radioactive Co.

The spectropolarimetry has already given exciting clues to the nature of the explosion. Something about the supernova is asymmetric. This will constrain the models, but more spectropolarimetry would be exceedingly valuable. The spectrum should be examined for clues as to whether it is the core or the outer envelope that is distorted.

Clearly the game of searching for and constraining the nature of any compact object 


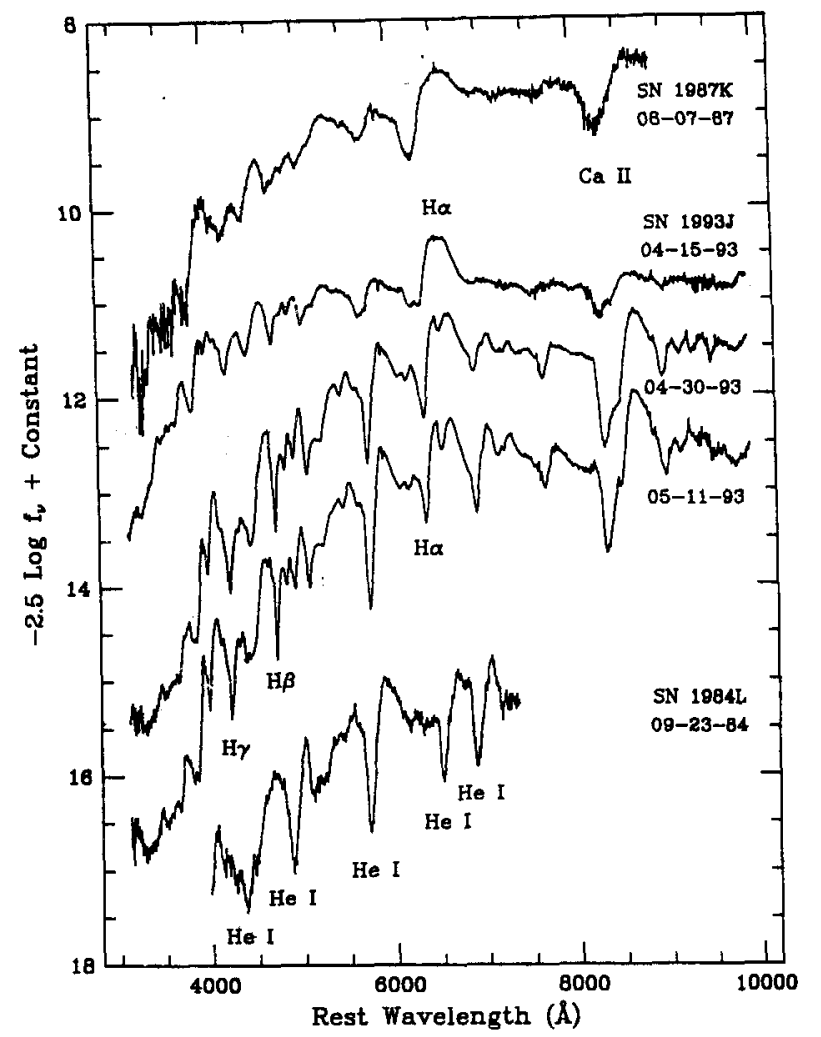

FIgURE 18. Spectra of SN 1993J in comparison with SN 1987K and SN Ib 1984L (Filippenko et al. 1993a).

in SN 1993J must be played out. The second peak of SN 1993J is similar in width to that of Type Ia, Ib, and Ic supernovae. SN 1993J is predicted to decline more slowly on the tail than models with comparable mass but no extended envelopes because the core/envelope interaction slows the expansion of the core and traps the $\gamma$-rays longer; however, the light curve is flatter on the tail than most of the models predict. Whether this is just the result of dynamics or radiative transfer, or requires an additional source of power, is not clear.

SN $1993 J$ is destined to provide considerably more physical insight into the taxonomy of supernovae. Figure 18 gives a sample of Lick spectra compared to the related events SN 1987K and SN 1984L. SN 1987K made a critical transition from a hydrogen-rich spectrum near maximum to a hydrogen-poor spectrum in the nebular phase. SN 1993J shows that this was not a unique event. There are significant differences in the details of the spectral profiles, but it is clear that SN 1993J and SN $1987 \mathrm{~K}$ are very closely related. Careful inspection of the spectra of SN 1987K published by Filippenko (1988) reveals, in hindsight, the first hints of the onset of the He I lines that occurred so markedly in SN 1993J. Note in Figure 18 that the He I lines in SN 1993J are very similar to those in the well-studied SN Ib 1984L. Arguments by analogy with other supernovae and based on quantitative spectral modeling predict that the $\mathrm{H}$ and $\mathrm{He}$ lines should fade in the optical 
at about 100 days and that the spectrum should be dominated by strong emission lines of $[\mathrm{O} \mathrm{I}],[\mathrm{Ca} \mathrm{II}]$, and $\mathrm{Ca}$ II by 200 days.

SN 1993J strengthens the hypothesis that Type Ib and Ic supernovae are triggered by core collapse in massive, evolved stars that have lost their outer envelope of hydrogen (Ib) and perhaps helium as well (Ic), often, but perhaps not always, through mass transfer in close binary systems. These objects are fundamentally related to Type II supernovae. Further study of SN 1993J should provide more insight into the evolutionary processes that lead to the various observed endpoints.

\section{Acknowledgments}

The contents of this review draw heavily on the plagiarized original contributions and draft submissions of the individuals and groups that presented results at the meeting. We are grateful for their contributions and hope that this summary does their work justice.

We would also like to acknowledge the special contribution made by the astronomers associated with the Isaac Newton group of telescopes that will greatly aid the general accessibility of data on supernovae. The supernova data obtained on La Palma are being reduced at the Royal Greenwich Observatory, and then placed on disk for rapid access by the community over the Internet. Descriptions of how the data have been reduced reside within the same disk areas as the data and, where appropriate, include details of the compromises that have been made. The reduced data are available via Internet using anonymous ftp at the address ftp.ast.cam.ac.uk. Any authors making use of the archive data in a publication should acknowledge the Isaac Newton Group of telescopes. The contact astronomer on La Palma for SN 1993J observations is Nic Walton (e-mail: naw"at"ing.iac.es).

The research of JCW is supported in part by NSF grant AST-9218035, NASA grants NAGW 2905 and GO 2563, and a grant from the R. A. Welch Foundation. AVF is supported by NSF grants AST-8957063 and 9115174, and by NSF Cooperative Agreement AST-8809616 (Center for Particle Astrophysics). We are grateful for the able help of Betty Friedrich in producing the $\mathrm{T}_{\mathrm{E}} \mathrm{X}$ document.

\section{REFERENCES}

Aldering, G.S., Humphreys, R.M., Richmond, M.W. (1993). AJ, 107, 662.

Andrillat, Y. (1993). IAU Circ. 5736.

Baron, E. (1993). Private communication.

Baron, E., Hauschildt, P.H., Branch, D., Wagner, R.M., Austin, S.J., Filippenko, A.V., Matheson, T. (1993). ApJ, 416, L21.

Benetti, S., Contarini, G., Gratton, R., Turatto, M. (1993). IAU Circ. 5751.

Bjorkman, K., Nordsieck, K. (1993). Private communication.

Blakeslee, J., Tonry, J. (1993). IAU Circ. 5758.

Blöcker, T., Schönberner, D. (1991). A\&A, 244, L43.

Brown, J.C., McLean, I.S. (1977). $A \& A$, 57, 141.

Chevalier, R.A. (1982). $A p J, \mathbf{2 5 9}, 302$.

Chevalier, R.A. (1992). $A p J$, 394, 599.

Chevalier, R.A., Fransson, C. (1994). ApJ, 420, 268.

Chipman, E. (1993). Compton Obs. Science Report \#132, August 12.

de Vaucouleurs, G. (1993, The Astronomer, 29, 278

de Vaucouleurs, G., Corwin, H.G., Jr., Skiff, B.A. (1994). PASP, 106, 156 
de Vaucouleurs, G. de Vaucouleurs, A., Corwin, H.G., Jr., Buta, R.J., Paturel, G., Fouqué, P. (1991). Third Reference Catalogue of Bright Galaxies (New York: Springer-Verlag).

Eastman, R.G. (1993). Private communication.

Filippenko, A.V. (1988). AJ, 96, 1941.

Filippenko, A.V. (1993a). IAU Circ. 5737.

Filippenko, A.V. (1993b). Private communication.

Filippenko, A.V., Matheson, T. (1993a). IAU Circ. 5740.

Filippenko, A.V., Matheson, T. (1993b). IAU Circ. 5787.

Filippenko, A.V., Matheson, T., Ho, L.C. (1993a). ApJ, 415, L103.

Filippenko, A.V., Matheson, T., Richmond, M.W., Treffers, R.R., Leibundgut, B., Paik, Y. (1993b). Workshop contribution.

Fox, G. K. (1991). ApJ, 379, 663.

Fransson, C. (1984). AA, 133, 264.

Freedman, W.L., et al. (1994). ApJ, 427, 628.

Hashimoto, M., Iwamoto, K., Nomoto, K. (1993). ApJ, 414, L105.

Hauschildt, P.H. (1992a). JQSRT, 47, 433.

Hauschildt, P.H. (1992b). ApJ, 398, 224.

Hauschildt, P.H. (1993). JQSRT, 50, 301.

Hauschildt, P.H., Baron, E. (1993). In preparation.

Hö̈lich, P., Müller, E., Khokhlov, A. (1993a). $A \& A$, 268, 570.

Höflich, P., Langer, N., Duschinger, M. (1993b). $A \& A$, 275, L29.

Hu, J.Y., Li, Z.W., Jiang, X.J., Wang, L.F. (1993). IAU Circ. 5777.

Humphreys, R.M., Aldering, G.S., Bryja, C.O., Thurmes, P.M. (1993). IAU Circ. 5739.

Iglesias, C.A., Rogers, F.J., Wilson, B.G. (1992). ApJ, 397, 717.

Jacoby, G.H., Ciardullo, R., Ford, H.C., Booth, J. (1989). ApJ, 344, 704.

Jannuzi, B., Schmidt, G., Elston, R., Smith, P. (1993). IAU Circ. 5776.

Jeffery, D., Branch, D. (1990). In Supernovae, ed. J.C. Wheeler, T. Piran, S. Weinberg (Singapore: World Scientific), p. 149.

Jeffery, D., et al. (1994). ApJ, 421, L27.

Khokhlov, A. (1993). Private communication.

Kurucz, R.L. (1991). In Stellar Atmospheres: Beyond Classical Models, ed. L. Crivellari, I. Hubeny, D.G. Hummer (Dordrecht: Kluwer), p. 440.

Langer, N. (1991). $A \& A, 252,669$.

Liebert, J. (1993). Private communication.

Lewis, J., et al. (1993). Workshop contribution.

Lundqvist, P., Fransson, C., Chevalier, R.A. (1993). Workshop contribution.

Lundqvist, P., Fransson, C. (1988). $A \& A, 192,221$.

Meikle, P., et al. (1993). Gemini, 40, 1.

Miller, J.S. (1993). Private communication.

Morrison, L.V., Argyle, R.W., Helmer, L. (1993). IAU Circ. 5767.

Nieuwenhuijzen H., de Jager, C. (1990). A\&A, 231, 134.

Nomoto, K., Susuki, T., Shigeyama, T., Kumagai, S., Yamaoka, H., Saio, H. (1993). Nature, 364, 507.

Ostriker, J.P. (1987). Nature, 327, 287.

Perelmuter, J.M. (1993). IAU Circ. 5736.

Podsiadlowski, Ph., Joss, P.C., Hsu, J.J.L. (1992). ApJ, 391, 246.

Podsiadlowski, Ph., Hsu, J.J.L., Joss, P.C., Ross, R.R. (1993). Nature, 364, 509. 
Pooley, G.G., Green, D.A. (1993). MNRAS, 264, L17.

Prugniel, P. (1993). IAU Circ. 5742.

Rathnasree, N., Ray, A. (1992). JA\&A, 13, 3.

Ray, A., Singh, K.P., Sutaria, F.K. (1993). JA\&A, 14, 53.

Richmond, M.W. (1993). IAU Circ. 5739.

Richmond, M.W., Treffers, R.R., Filippenko, A.V., Paik, Y., Leibundgut, B., Schulman, E., Cox, C.V. (1994). AJ, 107, 1022.

Ripero, J. (1993). IAU Circ. 5731.

Rots, A.H., Shane, W.W. (1975). $A \mathscr{B} A, 45,25$.

Rybicki, G.B., Hummer, D.G. (1991). $A \mathscr{E} A, \mathbf{2 4 5}, 171$.

Salzer, J., Herbst, W., Vinton, G. (1993). IAU Circ. 5741.

Schmidt, B.P. (1993). Private communication.

Schmidt, B.P., et al. (1993). Nature, 364, 600.

Shapiro, P.R., Sutherland, P.G. (1982). ApJ, 263, 902.

Shigeyama, T., Nomoto, K., Tsujimoto, T., Hashimoto, M. (1990). A\&A, 361, L23.

Shigeyama, T., Suzuki, T., Kumagai, S., Nomoto, K., Saio, H., Yamaoka, H. (1994). ApJ, 420, 341.

Smith, P. (1993). IAU Circ. 5776.

Smith, V.V., Tomkin, J. (1993). Private communication.

Sonneborn, G., Rodriguez, P.M., Wamsteker, W., Fransson, C., Kirshner, R.P. (1993). IAU Circ. 5754.

Steinmetz, M., Höflich, P. (1992). $A \& A, 257,641$.

Suntzeff, N.B., et al. (1988). $A J, \mathbf{9 6}, 1864$.

Suzuki, T., Kumagai, S., Shigeyama, T., Nomoto, K., Yamaoka, H., Saio, H. (1994). ApJ, 419, L73.

Swartz, D.A., Filippenko, A.V., Nomoto, K., Wheeler, J.C. (1993a). ApJ, 411, 313.

Swartz, D.A., Clocchiatti, A., Benjamin, R., Lester, D.F., Wheeler, J.C. (1993b,). Nature, 365, 232.

Tanaka, Y., et al. (1993). IAU Circ. 5753.

Taniguchi, Y., et al. (1993). PASJ Lett., 45, L43.

Tomaney, A., Crotts, A. (1993). IAU Circ. 5771.

Tonry, J.L. (1991). ApJ, 373, L1.

Trammell, S.R., Dinerstein, H.L., Goodrich, R.W. (1993). ApJ, 402, 249.

Trammell, S.R., Hines, D.C. (1993). Private communication.

Trammell, S.R., Hines, D.C., Wheeler, J.C. (1993). $A p J$, 414, L21.

Tran, H.D., Filippenko, A.V. (1993). Private communication.

Tuchman, Y., Wheeler, J.C. (1989). ApJ, 346, 417.

Utrobin V. (1993). Workshop contribution.

Van Driel, W., et al. (1993). PASJ Lett., 45, L59.

Van Dyk, S.D., Weiler, K.W., Rupen, M.P., Sramek, R.A., Panagia, N. (1993a). IAU Circ. 5759.

Van Dyk, S.D., Weiler K.W., Sramek R.A., Rupen, M.P., Panagia, N. (1993b). Workshop contribution.

Wamsteker W., Rodriguez P.M., Gonzalez R., Sonneborn G., Kirshner, R.P. (1993). IAU Circ. 5738.

Weiler, K.W., Panagia, N., Sramek, R.A. (1990). ApJ, 364, 611.

Wells, L.A., et al. (1993). Submitted, and workshop contribution.

Wheeler, J.C. (1993). SN computer network communication, June 7. 
Wheeler, J.C., et al. (1993). ApJ, 417, L71 .

Woosley, S.E. 1991, in Supernovae, ed. S.E. Woosley (New York: Springer-Verlag), p. 202.

Woosley, S.E., Eastman, R.G., Weaver, T.A., Pinto, P.A. (1994). ApJ, 429, 300.

Woosley, S.E., Pinto, P.A., Martin, P.G., Weaver, T.A. (1987). ApJ, 318, 664.

Zimmermann, H.-U., et al. (1993a). IAU Circ. 5748; (1993b). IAU Circ. 5750; (1993c). IAU Circ. 5766. 